\title{
OPEN \\ Molecular landscape of etioplast inner membranes in higher plants
}

\author{
Davide Floris $₫ \bowtie$ and Werner Kühlbrandt $₫ \bowtie$
}

\begin{abstract}
Etioplasts are photosynthetically inactive plastids that accumulate when light levels are too low for chloroplast maturation. The etioplast inner membrane consists of a paracrystalline tubular lattice and peripheral, disk-shaped membranes, respectively known as the prolamellar body and prothylakoids. These distinct membrane regions are connected into one continuous compartment. To date, no structures of protein complexes in or at etioplast membranes have been reported. Here, we used electron cryo-tomography to explore the molecular membrane landscape of pea and maize etioplasts. Our tomographic reconstructions show that ATP synthase monomers are enriched in the prothylakoids, and plastid ribosomes in the tubular lattice. The entire tubular lattice is covered by regular helical arrays of a membrane-associated protein, which we identified as the 37-kDa enzyme, light-dependent protochlorophyllide oxidoreductase (LPOR). LPOR is the most abundant protein in the etioplast, where it is responsible for chlorophyll biosynthesis, photoprotection and defining the membrane geometry of the prolamellar body. Based on the 9-A-resolution volume of the subtomogram average, we propose a structural model of membrane-associated LPOR.
\end{abstract}

D uring the past decade, electron cryo-tomography (cryo-ET) combined with subtomogram averaging has developed into a powerful technique for analysing the structure of biological assemblies in cells and organelles. In chloroplasts from higher plants $^{1}$ and algae ${ }^{2}$, cryo-ET has proved to be particularly efficient for direct identification of protein complexes in mature thylakoid membranes. Electron microscopy of chloroplast precursors, known as etioplasts, has to date been limited to chemically fixed and resin-embedded material. However, neither treatment conserves the high-resolution detail required for identification of complexes or structure determination in native membranes ${ }^{3-8}$.

Etioplasts are well suited to the study of chloroplast development by cryo-ET. In flowering plants (angiosperms) the organelles, which are photosynthetically inactive, accumulate in etiolated seedlings grown under low light. Etioplasts are characterized by a unique inner membrane network consisting of an extensive paracrystalline mesh of tubular membranes, the prolamellar body, attached to thylakoid precursors, the prothylakoids ${ }^{9}$. Prothylakoids appear as disk-like planar membranes in plastic sections of leaf material or purified intact etioplasts ${ }^{4-6,8,10}$, but assume a characteristic vesicular shape when isolated from inner membrane fractions ${ }^{3,11-15}$. Following light exposure, the whole system undergoes a profound metamorphosis associated with the gradual assembly of photosynthetic membrane protein complexes and the formation of interconnected grana stacks $5,6,8$.

Numerous studies on the protein composition of membrane fractions isolated from etioplasts of Arabidopsis ${ }^{4}$, barley ${ }^{16}$, maize ${ }^{17}$, pea $^{5}$, pine ${ }^{18}$ and wheat ${ }^{3,19}$ show that light-dependent protochlorophyllide oxidoreductase (LPOR) is probably one of the most abundant membrane-associated proteins, highly expressed in the prolamellar body $\mathrm{y}^{3,19,20}$.

LPOR is among a handful of enzymes that are known to require light for catalysis ${ }^{21}$. Under physiological conditions, LPOR forms a photoactive complex with protochlorophyllide and nicotinamide adenine dinucleotide phosphate (NADPH). Light exposure triggers the reduction of protochlorophyllide to chlorophyllide, which immediately diffuses to the chlorophyll synthase where the $\mathrm{C} 17$ propionate is esterified with phytol as the final step in chlorophyll biosynthesis ${ }^{22,23}$. The photoactive complex has a photoprotective role in vitro, preventing the excited triplet state of protochlorophyllide from generating singlet oxygen ${ }^{24}$. Photosynthetic organisms producing chlorophyll in the absence of light rely on the phylogenetically unrelated dark-operative protochlorophyllide oxidoreductase ${ }^{25}$.

After extensive biochemical characterization ${ }^{26-28}$ Zhang et al. ${ }^{21}$ recently reported the first X-ray structures of LPOR from Synechocystis sp. and Thermosynechococcus elongatus. Even though some flexible domains are lacking, the structures suggest an overall similarity of LPOR with members of the short-chain dehydrogenase and reductase family, characterized by a central $\beta$-sheet surrounded by six main $\alpha$-helices. However, little is known about the location, membrane anchoring and oligomeric state of $\mathrm{LPOR}^{5,29,30}$, which limits our understanding of how the enzyme functions during photomorphogenesis. LPOR has been suggested to determine the shape, preserve the architecture and control the size of the prolamellar body $^{31-35}$, but the structural basis of these processes is unknown. Only traces of LPOR appear to be present in prothylakoids, which instead are thought to be populated by the second-most abundant complex in etioplast inner membranes, ATP synthase ${ }^{3}$.

In the present work, we explore the molecular landscape of etioplasts in higher plants. Building on previous studies where chemical fixation or other methodological limits prevented direct visualization and structure determination of protein complexes in native membranes, we integrate known morphological features of the etioplast inner membrane with new information about protein structure and distribution. Our tomographic volumes confirm the early accumulation of ATP synthase monomers in prothylakoids, which reflects the organization found in chloroplast stroma lamellae and grana end membranes ${ }^{1}$. Plastid ribosomes populate the stromal regions of the prolamellar body membrane lattice while its tubular membranes are enveloped by uniform, helical arrays of a small, membrane-associated protein. By a combination of biochemical analysis and subtomogram averaging, we identified this protein as 


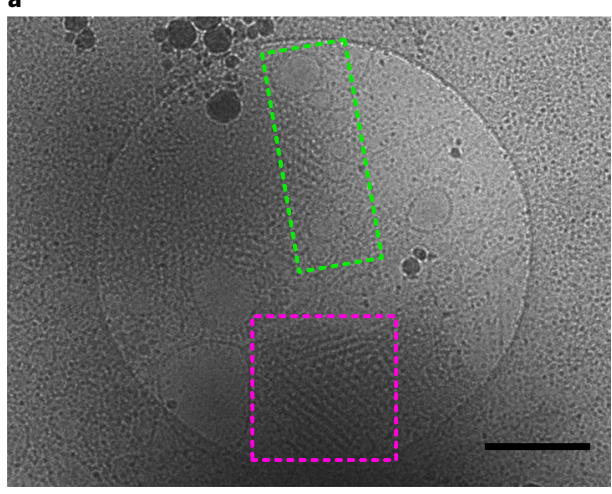

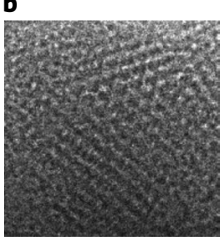

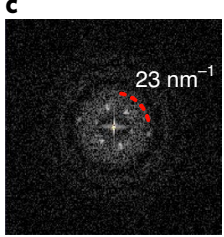

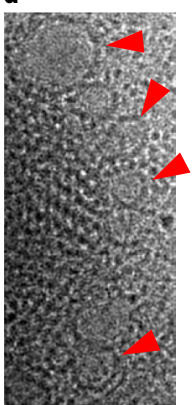

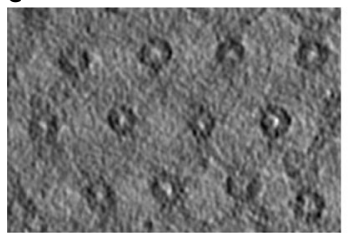

Slice 1

g

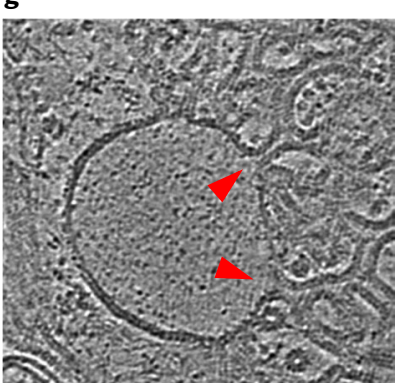

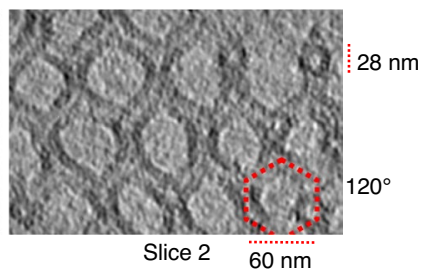

h

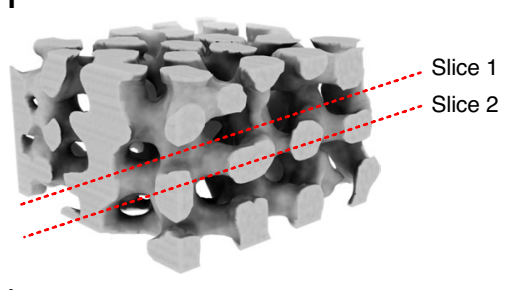

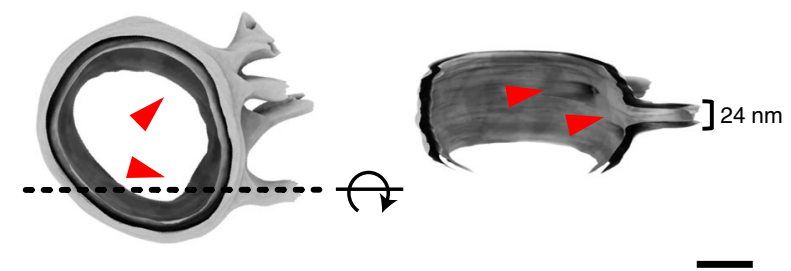

Fig. 1 Inner membrane morphology of pea etioplasts. a, Low-magnification view of vitrified inner membranes. b-d, Isolated patches of the micrograph in a (dashed magenta square and green rectangle) highlight the morphology of prolamellar bodies (b), their Fourier transform (c) and prothylakoids (d, red arrowheads). e,f, Tetrahedrally branched membrane tubes are evident in consecutive slices (e, slices 1 and 2 ) through the tomographic reconstruction of a prolamellar body and its segmented surface (f). $\mathbf{g}$-i, Tomographic section through a prothylakoid (g). Red arrowheads highlight circular junctions connecting the vesicular prothylakoid to the prolamellar body at positions indicated in the segmented surface (h) and rotated cross-section (i). Scale bars, $500 \mathrm{~nm}(\mathbf{a}), 50 \mathrm{~nm}(\mathbf{i})$.

the $37-\mathrm{kDa}$ enzyme LPOR and determine its membrane-associated structure at subnanometer resolution.

\section{Results}

Architecture of etioplast inner membranes. Because isolated intact plastids are too thick for imaging by cryo-tomography, we used small organelles that rupture during blotting for cryo-EM specimen preparation, which enabled us to record images of minimally disturbed inner membrane networks.

At low magnification, prolamellar bodies appear as a large, regular mesh of dense regions separated by a less dense stromal space (Fig. 1a). The high degree of order is clear in the Fourier transform of selected regions (Fig. 1b,c), which extends to the second order of diffraction $(\sim 1 / 23 \mathrm{~nm})$. The surrounding prothylakoids form large membrane vesicles (Fig. 1d) resembling those in plastic sections of isolated inner etioplast membranes ${ }^{3,11-15}$.

Tomographic reconstruction of minimally disturbed prolamellar bodies reveals the structure of their paracrystalline core, as seen in cross-sections at different heights of well-ordered areas (Fig. 1e,f). Membrane tubes measuring $\sim 28 \mathrm{~nm}$ in width intersect at an angle of $\sim 120^{\circ}$ to form tetrahedral units, with a repeat distance of $\sim 60 \mathrm{~nm}$. Their assembly results in a characteristic diamond cubic lattice.
The regularity of the prolamellar body reduces gradually towards its periphery, where multiple tubes connect to the same prothylakoid through $\sim 24-\mathrm{nm}$ circular junctions (Fig. 1g,h). In most cases the two membrane domains are adjacent; when further apart, they are connected by single, long membrane tubes (Extended Data Fig. 1a). The free ends of tubular membranes are sealed (Extended Data Fig. 1b), creating a closed membrane compartment that encompasses the entire etioplast inner membrane. Small spherical densities that appear to be plastoglobuli are often found in the outermost leaflet of both tubules and prothylakoids (Extended Data Fig. 1c,d).

Ribosomes and membrane-associated complexes. Numerous large protein complexes are visible on the internal membranes of ruptured etioplasts (Fig. 2a). The largest of these are $\sim 25 \mathrm{~nm}$ dense globular particles located in the stromal space of the tubular lattice (Fig. 2a,b, magenta arrowheads). A subtomogram average map at $\sim 26-\AA$ resolution identifies them as fully assembled ribosomes (Fig. 2e and Supplementary Fig. 1).

We fitted 50S (Protein Data Bank (PDB), 5mmi) and 30S (PDB, $5 \mathrm{mmj}$ ) subunits of chloroplast ribosomes as rigid bodies into the subtomogram averaging map (Extended Data Fig. 2a). Both subunits correlate well with the density, including the flexible foot region where chlororibosome-specific subunits cS22 and cS23 are 

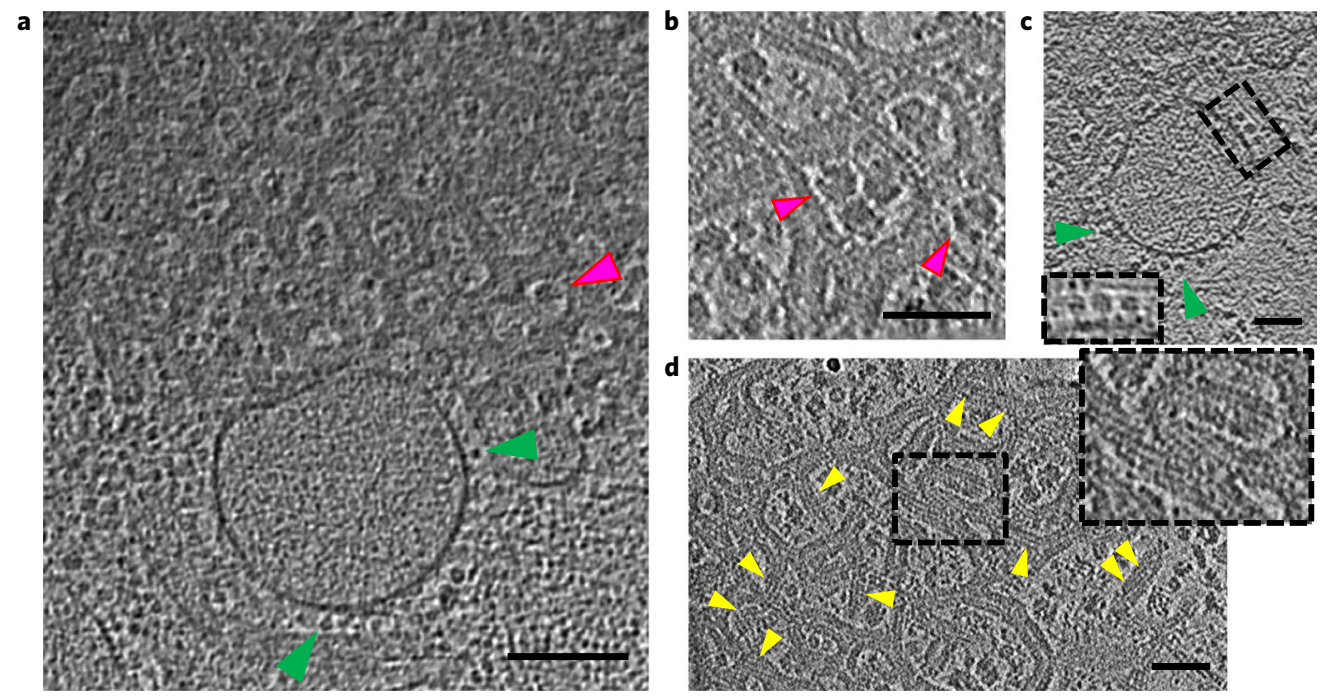

Local ice thickness, $230 \mathrm{~nm}$

Local ice thickness, $\sim 60 \mathrm{~nm}$

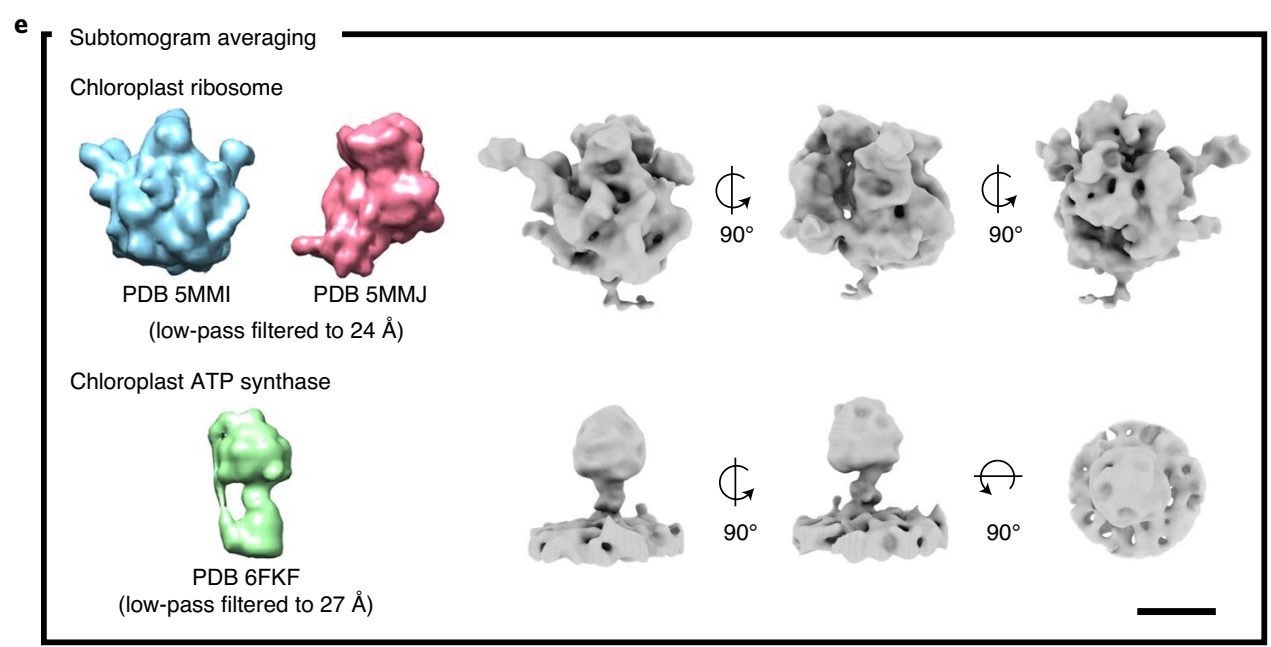

Fig. 2 | Protein complexes of prolamellar bodies and prothylakoids. a-e, Tomographic sections and subtomogram average maps. Dense globular particles $(\sim 25 \mathrm{~nm}$, magenta arrowheads in $\mathbf{a}, \mathbf{b})$ are fully assembled ribosomes (e, top row). Lollipop-shaped particles (green arrowheads in a,c) are ATP synthase (e, bottom row). Left-handed helical arrays (d, yellow arrowheads and inset) decorate the tubular membranes of the prolamellar body. Scale bars, $100 \mathrm{~nm}$ (a), $50 \mathrm{~nm}(\mathbf{b}-\mathbf{d}), 10 \mathrm{~nm}(\mathbf{e})$.

located (Extended Data Fig. 2b). In addition, we identified three conspicuous densities that have no correspondence in the atomic models of the chlororibosome. These regions are adjacent to subunits $\mathrm{uL} 10 \mathrm{c}$ and $\mathrm{bS} 1 \mathrm{c}$, and the polypeptide exit site (Extended Data Fig. 2c-e).

The second-largest complex we identified is a membrane protein with a diameter of $\sim 12 \mathrm{~nm}$, extending $\sim 16 \mathrm{~nm}$ above the stromal side of prothylakoid membranes (Fig. 2a,c, green arrowheads and inset). Subtomogram averaging yielded a map revealing the structure of chloroplast ATP synthase (PDB, 6fki) at $\sim 30-\AA$ resolution (Fig. 2e, Supplementary Fig. 1 and Extended Data Fig. 3a). ATP synthase distribution on the surface of prothylakoids appeared random, without formation of dimers or higher oligomers (Extended Data Fig. 3b). Similar densities are often visible on the stromal side of tubular membranes in prolamellar bodies (Extended Data Fig. 3c), but the prominent features of the surrounding membrane tubes precluded any meaningful subtomogram averaging.

Large numbers of a smaller, membrane-associated protein were identified on the membrane tubes of the paracrystalline meshwork (Fig. 2d, yellow arrowheads and inset, and Extended Data Fig. 4).
This protein was most clearly visible where the mesh was squeezed into a thin ( 100-nm) slab of vitrified buffer (Fig. 2d and Extended Data Fig. 1e), when it became apparent that the protein was arranged in a striking pattern of well-ordered helical arrays surrounding the tubular membranes. No such arrangement was evident in prothylakoids (Fig. 2c and Extended Data Fig. 3b).

Investigating the structure and identity of this protein in pea etioplasts was not straightforward. First, because of the small size of pea leaves, it is difficult to process sufficient plants for biochemical analysis of etioplast inner membranes in a short time; and second, the convoluted shape of appressed pea prolamellar bodies (Fig. 2d and Extended Data Fig. 1e) precluded subtomogram averaging.

Maize (Zea mays L.) etioplasts, on the other hand, have more desirable properties for both types of analysis. Etiolated maize seedlings produce larger leaves, and their prolamellar bodies have been reported to form straight, $\sim 26$-nm-wide tubules in response to mechanical stress. The same effect is induced by acidification below pH6.5 and high salt content ${ }^{15}$.

To determine whether the surface forces acting on etioplast membranes in the thin aqueous film during cryo-EM grid preparation 


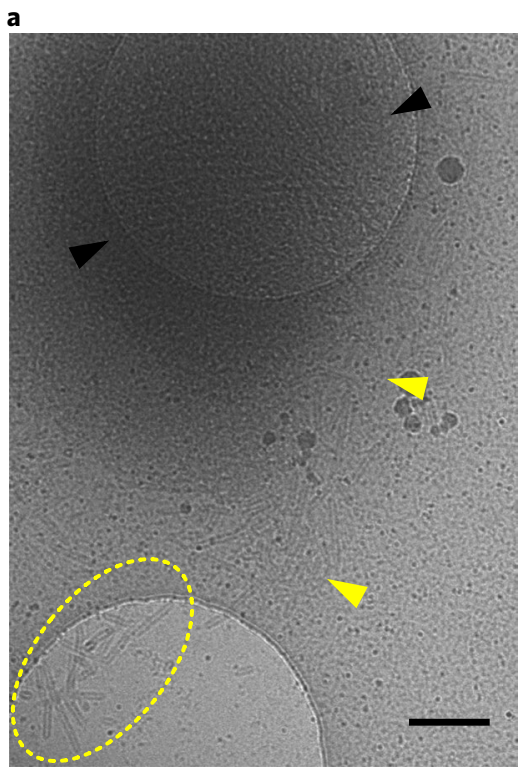

b
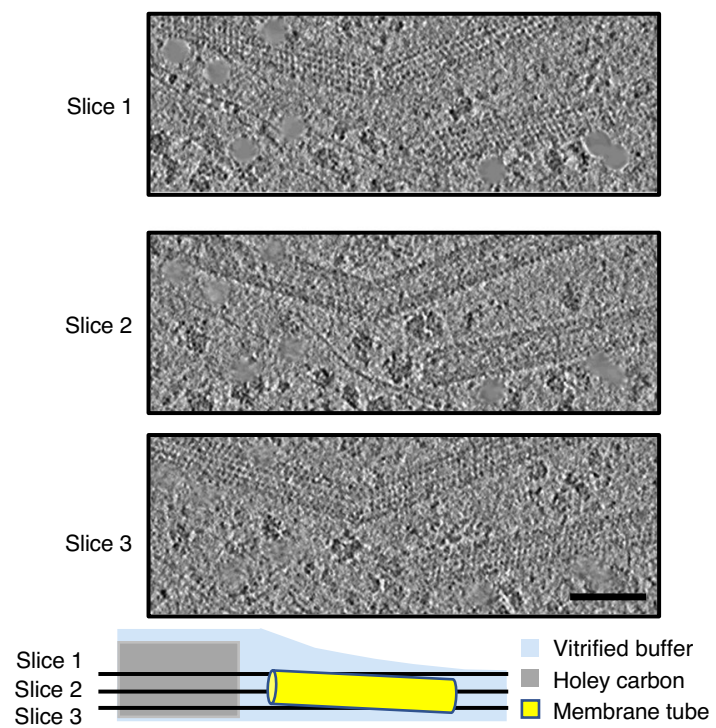

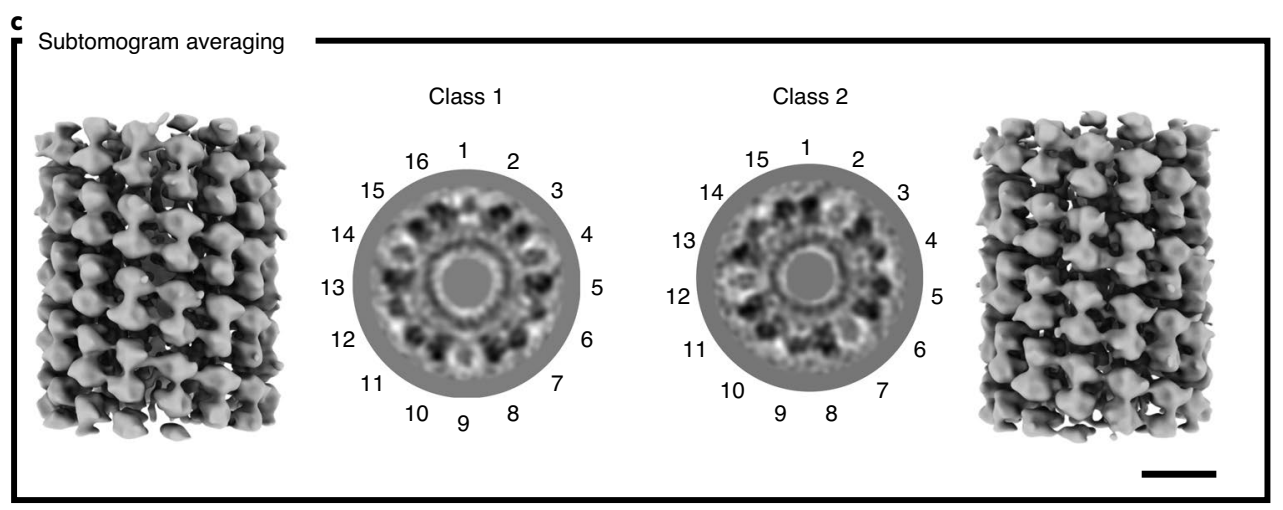

Fig. 3 | Helical arrays on membrane tubules. a, Overview at low magnification of compressed maize prolamellar bodies. Black arrowheads indicate the paracrystalline core of the prolamellar body; yellow arrowheads highlight a trail of straight tubular membranes connecting the core to a detached cluster (yellow dashed line). b, Consecutive slices through tomographic reconstruction (slices 1-3) show left-handed helical arrays identical to those in pea (Fig. 2d).c, Subtomogram averaging shows rows of dimers of a small, membrane-associated protein forming a left-handed helical lattice with either 16 (class 1) or 15 (class 2) units per turn, as evident in central cross-sections and lateral surface representations. Scale bars, $250 \mathrm{~nm}$ (a), $50 \mathrm{~nm}$ (b), $10 \mathrm{~nm}$ (c).

are sufficient for production of such elongated tubes, we repeated the cryo-EM grids with purified maize etioplasts. Possible effects of acidic $\mathrm{pH}$ and high salt were excluded by use of a low-salt, pH 8.0 buffer (Methods) previously shown to yield stable etioplast preparations $^{19}$.

Helical crystals of protochlorophyllide oxidoreductase cover prolamellar body membranes. Unperturbed inner membranes from maize etioplasts exhibit the same features as observed in pea (Extended Data Fig. 5). However, the tubules extending from compressed maize prolamellar bodies tended to be more elongated and straight, with some becoming detached and forming small clusters in the surrounding areas (Fig. 3a, yellow arrowheads and dashed oval). Their outer membrane surface was decorated by a helical pattern (Fig. 3b), much clearer than but otherwise apparently identical to that seen in pea (Fig. 2d).

On a sucrose density gradient, homogenized maize etioplasts separated into a lighter band of inner membranes and a heavier pellet of intact organelles and starch (Extended Data Fig. 6a). SDSpolyacrylamide gel electrophoresis (SDS-PAGE) of the lighter fraction indicated the presence of one predominant species with an apparent molecular weight of $\sim 37 \mathrm{kDa}$ (Extended Data Fig. $6 \mathrm{~b}$ ); immunoblot analysis identified this band as LPOR. In the same way, the second-most abundant component detected in SDS gels was attributed to the $\alpha$ - and $\beta$-subunits of ATP synthase (Extended Data Fig. 6b), in agreement with previous reports ${ }^{3,19}$. Immuno-gold labelling of leaf plastic sections with anti-LPOR antibodies resulted in the accumulation of gold beads on the paracrystalline core of the prolamellar body, which were largely absent in the surrounding areas (Extended Data Fig. 6c), indicating that the protein lattice decorating the membrane tubes is indeed LPOR.

Subtomogram averaging shows that LPOR forms rows of dimers that wrap around the tubes in a left-handed, three-start helical lattice (Fig. 3c), with three parallel helical filaments of dimers and a 24-nm pitch (Extended Data Fig. 7). Multi-reference alignment classification detected small differences in helix diameter. A total of 1,678 subvolumes fell into two main classes, featuring particles with either 16 (class 1) or 15 (class 2) units per turn (Fig. 3c). The outer and inner helix diameter of class 1 was, respectively, 29 and $\sim 14 \mathrm{~nm}$, whereas in class 2 both dimensions were narrower by $\sim 2 \mathrm{~nm}$ (Extended Data Fig. $7 \mathrm{~b}$ ). The two reconstructions were refined independently to 18.2 - and 18.1 - $\AA$ resolution, respectively (Supplementary Fig. 2), and used as references to improve local alignment in subsequent rounds of refinement. 


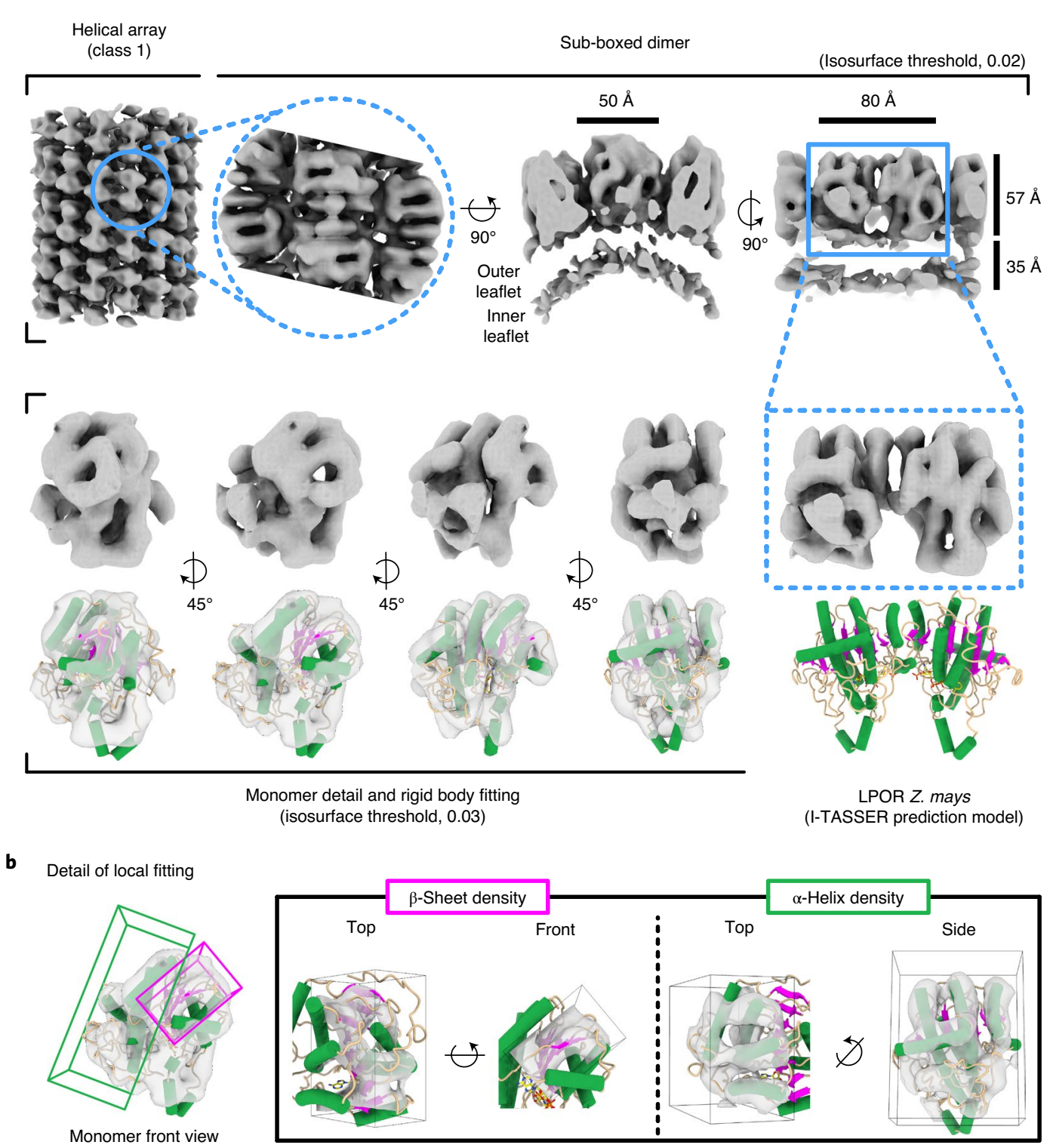

Fig. 4 | Structure of membrane-bound LPOR. a, Subtomogram average of class 1 sub-boxes. Top view and slice through side view indicate the size and orientation of the LPOR dimer on the membrane at an isosurface threshold of 0.02 . At a higher contour level (isosurface, 0.03), four different map views show the structural features of a single LPOR monomer and their close agreement with the model of maize LPOR generated by I-TASSER. $\mathbf{b}$, Close-up view of the elements of secondary structure. The $\beta$-sheet fits into a wide, flattened density; the $\alpha$-helices fit into elongated, rod-shaped densities.

Subnanometer-resolution structure of membrane-associated LPOR. Particles in classes 1 and 2 were sub-boxed along the helical path, then processed separately. Because the resulting maps were largely identical, it is sufficient to describe the model from class 1 only (Figs. 4 and 5 and Extended Data Fig. 8). LPOR forms symmetrical dimers with overall dimensions of $\sim 80 \times \sim 50 \AA$, extending to a height of $\sim 57 \AA$ above the membrane surface (Fig. $4 \mathrm{a}$ ). Slices along the helix axis indicate a negative (concave) curvature of the membrane where it interacts with the LPOR dimers (dashed red lines in Extended Data Fig. 8). The quality of the reconstructions, both at a resolution of $9 \AA$ (Extended Data Fig. 9), allows identification of partly resolved $\alpha$-helices and $\beta$-sheets (Fig. 4).

We used the programme I-TASSER ${ }^{36}$ to construct a three-dimensional (3D) model of LPOR based on the Zea mays primary sequence (Methods). Among multiple structural templates in the $\mathrm{PDB}$, the programme selected the X-ray structure of Synechocystis LPOR (PDB: 6r48 (ref. $\left.{ }^{21}\right)$ ) as the best fit and generated a template-based structural model (Fig. 4a and Extended Data Fig. 10).

The model of maize LPOR features a central, eight-stranded $\beta$-sheet surrounded by ten $\alpha$-helices (Extended Data Fig. 10a). A $\mathrm{NADPH}$ molecule was placed within the catalytic pocket (red circle in Fig. 5a and Extended Data Fig. 10a), because the X-ray structure of cyanobacterial LPOR shows NADPH bound in this position. The protochlorophyllide substrate was not modelled. Rigid-body fitting (Figs. 4 and 5a) indicates a high correlation factor of 0.9 between the in silico model and our subtomogram average density map. The $\beta$-sheet and most helices of the protein align vertically on top of the membrane (Figs. 4 and 5a and Extended Data Fig. 8). No transmembrane segment is evident. Anchoring to the outer leaflet of the bilayer appears to be mediated by helices 7 and 8 . 


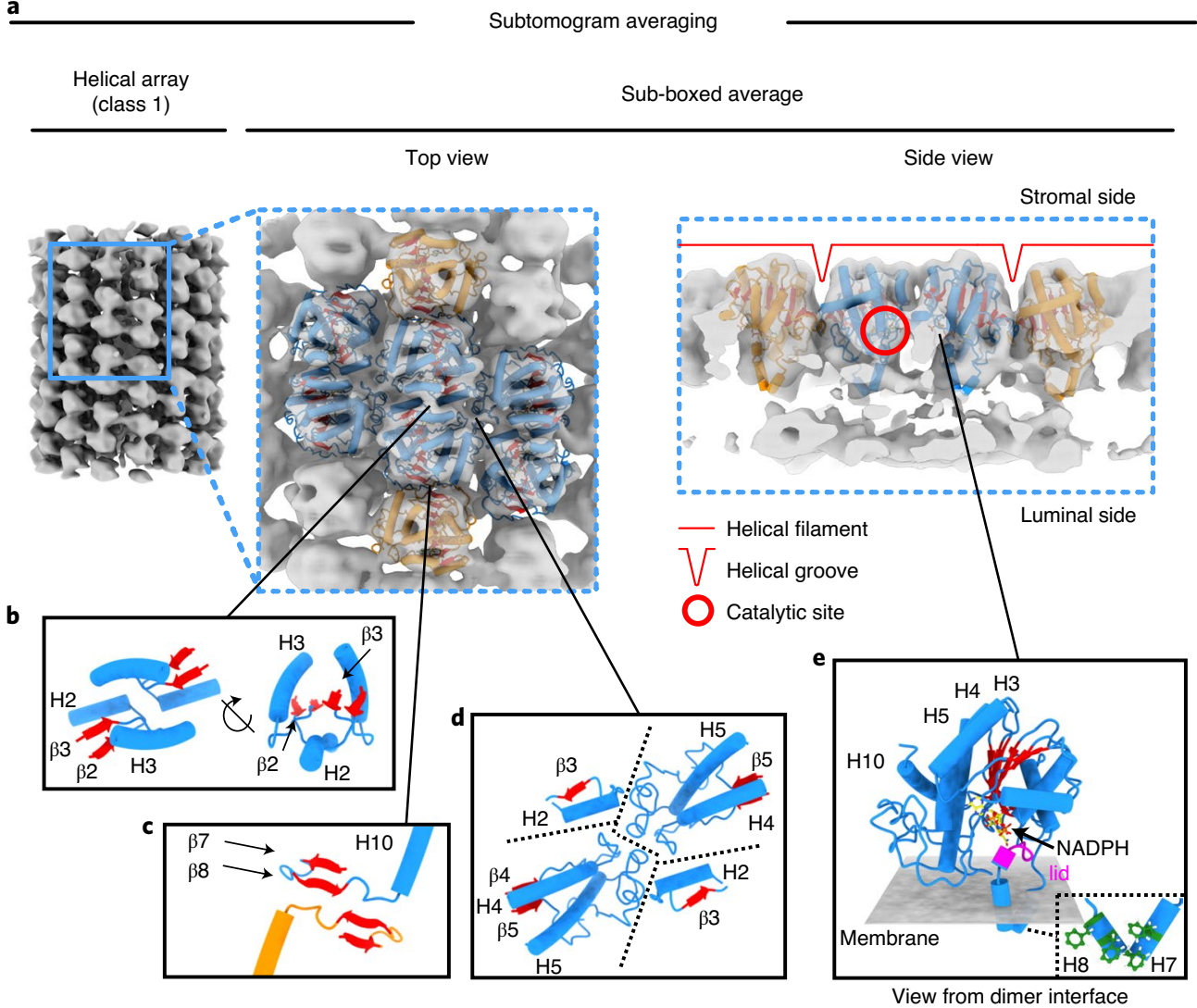

Fig. 5 | Membrane interaction and molecular contacts of LPOR monomers. a, Rigid-body fitting of maize LPOR model to the subtomogram average map. Blue models refer to the central helical filament, orange models to neighbouring filaments. b-d, interaction sites at the dimer interface (b), the interface between dimer rows (c) and between dimers (d). Dashed lines in $\mathbf{d}$ separate secondary structure elements from different monomers. $\mathbf{e}$, Detail of proteinmembrane interaction, with membrane surface in grey, lid domain (residues 246-252) in magenta and NADPH in yellow. The inset indicates the position of four Phe residues (green) likely to promote membrane interaction. $\mathrm{H}$, helix.

At the dimer interface, residues in the links from $\beta$-strand 2 to helix 2 and $\beta$-strand 3 to helix 3 closely approach the corresponding residues of the opposite monomer (Fig. 5b). The flexible regions linking $\beta$-strand 4 to helix 4 and $\beta$-strand 5 to helix 5 stretch sideways towards the long side of the next dimer, contacting as indicated in Fig. 5d. Minor contacts are also evident across dimers on neighbouring helical filaments, suggesting an interaction between segments joining $\beta$-strand 8 to helix 10 on either side of the helical groove (Fig. 5c).

\section{Discussion}

We used cryo-ET to study the supramolecular organization of protein complexes in frozen-hydrated inner membranes of etioplasts from pea and maize. The etioplast membrane systems were readily observable on EM grids when the organelles were disrupted by blotting immediately before plunge-freezing. This approach, introduced by Daum et al. ${ }^{1}$, preserves lateral membrane heterogeneity and native protein composition. Previous studies with plastic sections of chemically fixed organelles and leaves ${ }^{6-8}$, although unable to reveal molecular detail, are broadly consistent with our cryo-tomograms of unfixed, flash-frozen organelles. By contrast, our cryo-tomograms and subtomogram averages show the $3 \mathrm{D}$ structures of etioplast membrane protein complexes directly in the native membranes.

Prothylakoids are characterized by low curvature, absence of appressed regions and a high percentage of the bilayer-forming lipid digalactosyldiacylglycerol. In this respect they resemble chloroplast stroma lamellae and grana end membranes. Vesicular prothylakoids are frequently found in preparations of isolated etioplast inner membranes ${ }^{3,11,12,14,15}$. In our specimens (Fig. 1a and Extended Data Fig. 5a), this morphology is probably due to slight osmotic swelling before or during plunge-freezing, which is an unavoidable consequence of the reduced osmolarity of the buffers used for vitrification. A low concentration of osmolytes, such as sugars and salts, is mandatory to warrant sufficient contrast for cryo-EM analysis.

ATP synthase is distributed uniformly in prothylakoid membranes (Fig. 2a,c and Extended Data Fig. 3b). Its monomeric state, evident in our tomographic reconstructions (Extended Data Fig. 3b), is in line with cryo-tomographic studies of mature thylakoid membranes ${ }^{1}$ and native gel electrophoresis ${ }^{16}$. Occasional lollipop-shaped membrane protein complexes found in the tubular lattice of the prolamellar body (Extended Data Fig. 3c) suggest that this membrane region also contains some ATP synthase, consistent with an earlier biochemical analysis of isolated etioplast inner membrane fractions ${ }^{3}$.

From a functional perspective, the operational framework of etioplast ATP synthase is affected by the absence of a fully assembled photosynthetic electron transport chain. In a recent study, Kambakam et al. ${ }^{37}$ suggested that the chemiosmotic requirements for ATP synthesis in etioplasts may be satisfied by the proton-pumping activity of $\mathrm{NAD}(\mathrm{P}) \mathrm{H}$ dehydrogenase (NDH) and cytochrome $b_{6} / f$ $\left(\right.$ Cyt $\left.b_{6} / f\right)$. This is proposed as part of a larger pathway similar to mitochondrial oxidative phosphorylation, also found in other 
Dark

(skotomorphogenesis)

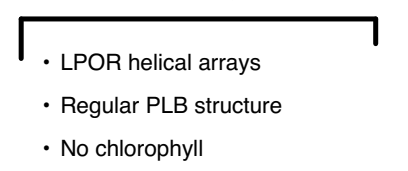

Light

(photomorphogenesis)

$$
\begin{aligned}
& \text { - LPOR arrays dissociate, expression decreases } \\
& \text { - PLB disassembles and thylakoids form } \\
& \text { - Chlorophyll concentration increases }
\end{aligned}
$$
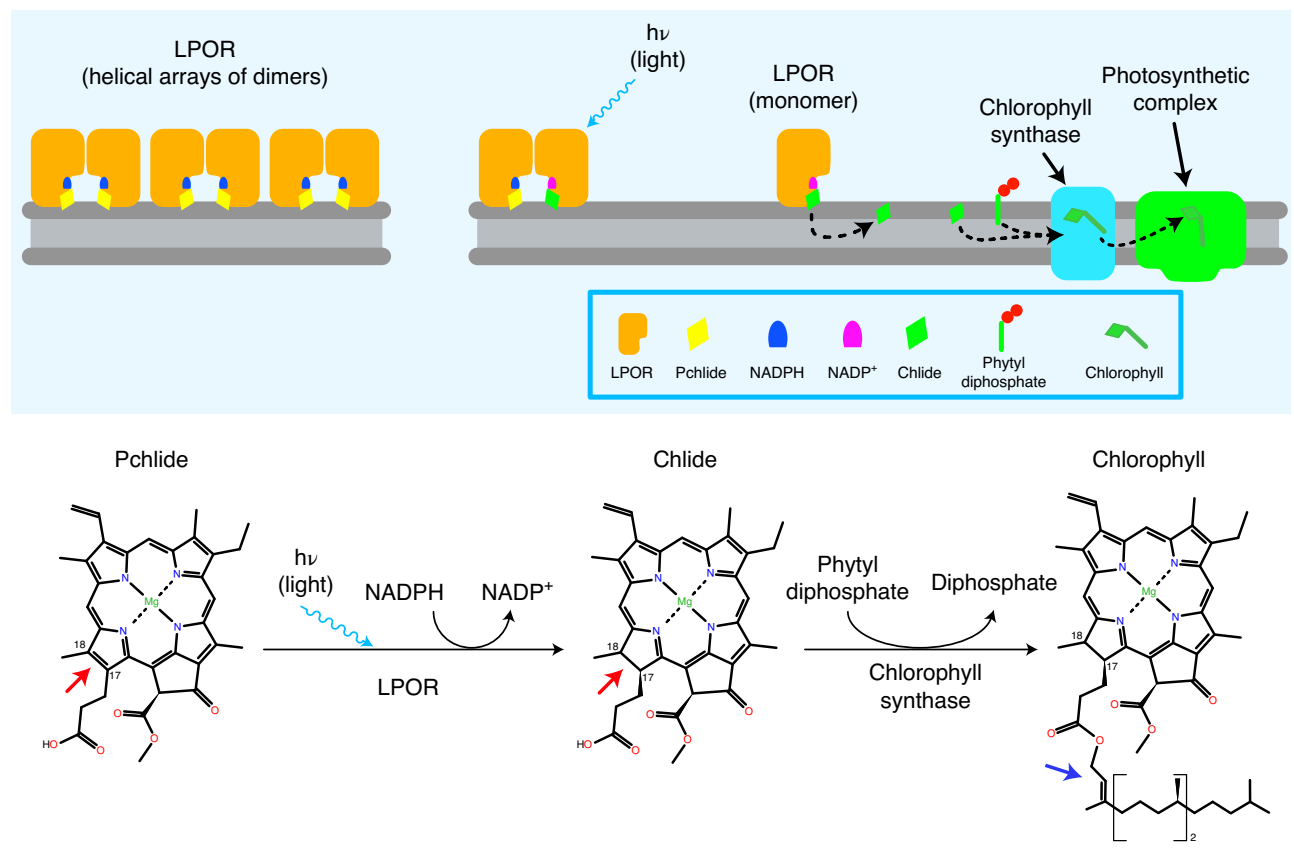

Fig. 6 | Proposed mechanism for chlorophyll biosynthesis in plastid membranes. Under dark conditions, helical LPOR arrays preserve the compact, regular architecture of the prolamellar body (PLB) while storing a ready-to-use pool of protochlorophyllide (Pchlide). Light exposure triggers LPOR activation and the PLB disassembles. LPOR converts Pchlide to chlorophyllide (Chlide), which diffuses in the membrane to the chlorophyll synthase. Chlide is esterified with a phytol chain to produce chlorophyll, which is immediately bound by a photosynthetic complex. Red arrows in the lower panel highlight the C17-C18 bond reduced by LPOR. The blue arrow indicates the position of the phytol chain esterified by the chlorophyll synthase.

photosynthetically inactive plastids (reviewed in Renato et al..$^{38}$ ). Apart from ATP synthase, none of the other protein complexes involved are resolved in our tomograms, most probably because of their lower molecular mass or the smaller size of their solvent-exposed domains.

Large globular densities are occasionally found in the stromal region within the tubular lattice of prolamellar bodies (Fig. 2a,b). Earlier work referred to these as 'ribosome-like particles', because they were degraded by ribonuclease ${ }^{11}$. Our subtomogram averaging map (Fig. 2e and Extended Data Fig. 2) provides direct evidence that they are mature chloroplast ribosomes. At the current resolution $(\sim 30 \AA)$, the three conspicuous density regions that are absent from the atomic model of Bieri et al..$^{39}$ cannot be identified unambiguously. Previous studies, however, report structures with similar features that may shed light on their identity and function.

The most prominent of these three regions is an arc-shaped density protruding from the $50 \mathrm{~S}$ uL10c subunit (Extended Data Fig. 2c). L7/L12 oligomers bound to bacterial ribosomes share the same feature ${ }^{40}$. These proteins form a flexible stalk that is thought to facilitate the recruitment of translation factors and guanosine triphosphate hydrolysis. The second region is attached to bS1c in the $30 \mathrm{~S}$ subunit (Extended Data Fig. 2d), a flexible protein that plays a central role in messenger RNA binding and translation initiation and is only partially resolved in the atomic model ${ }^{39}$. One of its oligonucleotide-binding folds protrudes towards the mRNA exit channel. The other two oligonucleotide-binding folds are not identified, and may account for some of the unassigned density in our subtomogram average (Extended Data Fig. 2d, dashed red line).
The third region is a small density near the polypeptide exit site (Extended Data Fig. 2e), similar to the ribosomal RNA loop that stabilizes membrane interaction during cotranslational translocation in bacterial ribosomes ${ }^{41}$. Further investigation should resolve the molecular details of these regions, and provide insight into the activation state and membrane association of etioplast ribosomes.

LPOR assembles into highly ordered helical arrays that decorate the surface of prolamellar bodies (Figs. $2 \mathrm{~d}$ and $3 \mathrm{~b}$ and Extended Data Fig. 4). This observation explains the direct correlation between the size of the paracrystalline membrane network and LPOR expression levels ${ }^{32-34}$. Additionally, it explains the recent findings of Yamamoto et al. ${ }^{42}$, who showed that overexpression of LPOR in etiolated cyanobacteria produces large membrane structures that look like prolamellar bodies. Isolated membrane tubes (Fig. 3 and Extended Data Fig. $7 \mathrm{~b}$ ) present the same diameter as those in the meshwork of the prolamellar body (Fig. 1e), as previously observed by Selstam et al. ${ }^{15}$. They appear, however, to be straighter and longer, possibly as a consequence of etioplast rupture during blotting. Further investigation under experimental conditions that do not generate mechanical stress (for example, by examining focused ion beam-milled lamellae of vitrified plant cells) should clarify these aspects.

By comparison, the diameter of prothylakoid junctions was narrower by $\sim 4 \mathrm{~nm}$ (Fig. 1g,h). This may signify a loss of order in the LPOR helical arrays, or a transition in protein composition between the two membrane domains. The regular decoration with LPOR protein is not evident in prothylakoids, but the presence of a smaller pool of less ordered complexes cannot be ruled out as these would be harder to identify. 
Experiments based on cross-linking, fluorescence and circular dichroism spectroscopy of both native membranes and reconstituted photoactive complexes have previously been performed, suggesting that LPOR might form large aggregates ${ }^{43-45}$, but nothing was known about their arrangement and order. Our cryo-ET results provide direct evidence of array formation in vivo and greatly expand the model proposed by Reinbothe and co-authors ${ }^{29,46}$, where heterologous expression and in vitro reconstitution with lipids yielded LPOR hexamers dubbed light-harvesting POR:protochlorophyllide supercomplexes.

According to our model, the residues that mediate monomer interaction at the dimer interface are probably located in helices 2-3, $\beta$-strands $2-3$ or the flexible loops connecting them (Fig. 5b). Deletion of the 'extra loop', a small region distinguishing LPOR from the structurally related alcohol dehydrogenases, was shown to impair LPOR oligomer formation ${ }^{47}$. This loop, corresponding to Gly166-Asp201 in maize LPOR, is located upstream of helix 5 and appears to mediate a lateral contact between adjacent dimers (Fig. 5d).

The effect of individual LPOR dimers on membrane morphology is evident from the local negative curvature observed in our subtomogram averages (Fig. 4a and Extended Data Fig. 8). Based on the configuration determined by rigid-body fitting of the predicted model from maize (Figs. 4 and 5a), we propose that helices 7 and 8 might stabilize interaction with the bilayer through four Phe residues (259, 263, 266 and 269; Fig. 5e). In cyanobacterial LPOR, the corresponding residues were suggested to be part of a large hydrophobic patch that plays a role in catalytic activity, protochlorophyllide binding and the formation of higher-order assemblies ${ }^{48}$.

Other than by protein interaction, negative membrane curvature might result from the physicochemical properties of monogalactosyldiacylglycerol (MGDG), the most abundant lipid in the prolamellar body ${ }^{12}$. MGDG has an intrinsic tendency to form inverse hexagonal lipid phases, which would create negative curvature and elastic stress, and exert lateral pressure on proteins embedded in the lipid bilayer. Recent evidence suggests that MGDG regulates LPOR allosterically, inducing conformational changes with a strong effect on catalytic efficiency ${ }^{45}$. Conversely, the absence of MGDG is detrimental to the formation and oligomerization of the photoactive LPOR complex ${ }^{10}$. Photoprotective carotenoids such as lutein might play an equally important role, since these are known to influence the morphology of the prolamellar body and its interaction with $\mathrm{LPOR}^{49}$.

In LPOR from cyanobacteria ${ }^{21}$ a loop between residues 223 and 229 acts as a lid for the catalytic site. In maize, this region corresponds to residues 246-252, which are part of helix 6 and the loop connecting it to $\beta$-strand 6 (magenta in Fig. 5e). Our analysis suggests that, in the physiological context of the prolamellar body, LPOR assumes an optimal orientation to facilitate the swift exchange of protochlorophyllide and chlorophyllide between its catalytic site and the outer membrane leaflet (Figs. 5e and 6). This would be in agreement with the amphipathic character of protochlorophyllide, which would make it compatible with the lipid head groups of the outer leaflet and promote its uptake from LPOR ${ }^{50,51}$.

Sequestration of protochlorophyllide by LPOR has been suggested to prevent generation of photo-oxidized toxic forms of the pigment ${ }^{22}$. The tight packing and uniform distribution of LPOR on the etioplast inner membranes leaves room for speculation about a more elaborate photoprotective system along the lines of a resonance-energy transfer mechanism between neighbouring LPOR complexes ${ }^{29,46}$. This might resemble the carotenoid- and chlorophyll-based photoprotective mechanisms that are well characterized in photosystems and light-harvesting complexes of mature thylakoids ${ }^{52}$.

\section{Methods}

Etioplast isolation. Pea and maize seedlings were grown in a darkened growth chamber at a constant temperature of $20^{\circ} \mathrm{C}$ for 7 and 13 days, respectively.
Etioplasts were isolated according to Blomqvist et al. ${ }^{19}$ with minor modifications. Briefly, leaves were ground with a Waring blender in ice-cold buffer A (pea: $50 \mathrm{mM}$ Tricine/ $\mathrm{NaOH}$ pH 7.5, $300 \mathrm{mM}$ sorbitol, $10 \mathrm{mM} \mathrm{CaCl}_{2}, 10 \mathrm{mM} \mathrm{MgCl}_{2}$; maize: $20 \mathrm{mM}$ HEPES/ $\mathrm{NaOH} \mathrm{pH} 8.0,300 \mathrm{mM}$ sorbitol, $10 \mathrm{mM} \mathrm{NaHCO}_{3}, 5 \mathrm{mM} \mathrm{MgCl}_{2}$ ), then filtered through four layers of Miracloth (Merck). The organelles were pelleted $\left(4,500 \mathrm{~g}, 15 \mathrm{~min}, 4^{\circ} \mathrm{C}\right)$, resuspended and further purified by centrifugation $(4,500 \mathrm{~g}$, $20 \mathrm{~min}, 4^{\circ} \mathrm{C}$ ) on a discontinuous $40-80 \%$ Percoll gradient (Merck) in buffer A. Intact etioplasts were isolated from the $40-80 \%$ interface and washed twice in buffer A to eliminate residual Percoll. All steps were carried out in the dark or under safe green light.

Biochemical analysis of etioplast inner membranes. Inner membranes were extracted from intact maize etioplasts by homogenization with a glass Potter homogenizer, then isolated from the $30-45 \%$ interface of a discontinuous $10 / 30 / 45 \%$ sucrose gradient in buffer A by centrifugation $\left(25,400 g, 2 \mathrm{~h}, 4^{\circ} \mathrm{C}\right)$ Protein composition was assessed by SDS-PAGE as previously described by Haniewicz et al. ${ }^{53}$, without modification. For immunoblot analysis, proteins were transferred on a polyvinylidene difluoride membrane with a Trans-Blot Turbo system and RTA Transfer Kit (Bio-Rad) according to the manufacturer's instructions. Anti-POR (no. AS05 067, dilution 1:2,000) and anti-ATP synthase (no. AS08 370, dilution 1:10,000) were used as primary antibodies (Agrisera), and goat anti-rabbit (no. 4055-05, dilution 1:1,000) as the secondary antibody (SouthernBiotech). The horseradish peroxidase signal was generated with the ECL Start Western Blotting Reagent kit (GE Healthcare) and detected with a ChemiDoc Touch Imaging System (Bio-Rad). Full scans of SDS-PAGE gel and immunoblots are available at Source Data Fig. 1.

For immuno-gold labelling, fresh sections of etiolated leaves were placed on a glass slide and gently sliced with a scalpel, then treated as described in Wilkes et al. ${ }^{54}$ with minor modifications. Briefly, after fixation with $4 \%$ paraformaldehyde in PBS, the sample was dehydrated in an ethanol-gradient series and infiltrated with LR white medium-grade acrylic resin (London Resin). After $48 \mathrm{~h}$ of polymerization at $65^{\circ} \mathrm{C}$, the pellets were cut into $60-80-\mathrm{nm}$ slices with an Ultracut S Microtome (Leica) and deposited on gold grids (Plano) coated with a layer of amorphous carbon and Formvar. Labelling was carried out with the same anti-POR primary antibody (dilution 1:200) as used for imunoblots, but gold-conjugated AffiniPure goat anti-rabbit (Jackson ImmunoResearch) was used as secondary antibody (no. 111-205-144, dilution 1:20). Samples were imaged in a Tecnai G2 Spirit BioTwin (FEI).

Cryo-EM grid preparation. Immediately before vitrification, fresh intact etioplasts were pelleted for $3 \mathrm{~min}$ with a benchtop centrifuge then resuspended in half the initial volume of cryo buffer (similar to buffer A, but with sorbitol replaced by $150 \mathrm{mM}$ trehalose). At this point the absence of chlorophyll, used as marker of accidental light exposure, was monitored by acetone extraction as described by Haniewicz et al. ${ }^{55}$. Organelles were mixed 3:1 with 6- or 10-nm gold fiducials (AURION Immuno Gold Reagents \& Accessories), then $3 \mu \mathrm{l}$ of suspension was immediately applied to glow-discharged Quantifoil R0.6/1 or R2/2 300 mesh copper grids (Quantifoil Micro Tools) and plunge-frozen with a Vitrobot Mark IV (FEI) after blotting for $6-8 \mathrm{~s}$ at $100 \%$ relative humidity.

Cryo-ET. Data were collected with a Titan Krios electron microscope (FEI) operated at $300 \mathrm{kV}$ in energy-filtered transmission electron microscopy mode, as previously described in D'Imprima et al. ${ }^{56}$. The magnification was chosen to achieve a calibrated pixel size of $2.2 \AA$. Tilt series were collected from $+60^{\circ}$ to $-60^{\circ}$ using a dose-symmetric acquisition scheme ${ }^{57}$ implemented in SerialEM ${ }^{58}$. The acquisition parameters are summarized in Supplementary Tab. 1. Dose-fractionated images were recorded in counting mode on a $\mathrm{K} 2$ summit or K3 direct electron detector (Gatan), then motion corrected and dose weighted with MotionCor2 (ref. ${ }^{59}$ ). Contrast transfer function correction and reconstruction with weighted back-projection were done in $\mathrm{IMOD}^{60}$. All tomographic images were slices ten pixels in thickness. Contrast was enhanced with either a nonlinear anisotropic diffusion filter ${ }^{61}$ or a 3D median filter in MATLAB (MathWorks). Volumes were segmented manually with a combination of the IMOD drawing tool plug-in and the EMAN convolutional neural network method ${ }^{62}$.

Subtomogram averaging and homology modelling. Subtomogram averaging was performed with Dynamo ${ }^{63}$ according to the gold standard procedure ${ }^{64}$. Chlororibosomes and ATP synthase subvolumes were extracted from seven tomograms. For the chlororibosome dataset, 315 particles within the tubular lattice of prolamellar bodies were picked manually while 377 ATP synthase complexes were selected from the neighbouring prothylakoids. Isolated ribosomes and ATP synthase complexes on membranes detached from prolamellar bodies were excluded from the analysis. In both datasets, 20 random particles were used as initial reference and the final maps were bandpass filtered to $26 \AA$ for ribosomes and $30 \AA$ for ATP synthase.

For LPOR averaging, membrane tubes from 17 tomograms were sampled at regular intervals along their axis with the addModPts function in PEET ${ }^{65}$ Multi-reference alignment, performed as in D'Imprima et al..$^{56}$, split them in two main classes of 950 and 728 particles featuring either 16 (class 1) or 15 (class 
2) helical repetitions per turn, respectively. Independent processing yielded reconstructions at 18.2- and 18.1-Å resolution. Both maps were bandpass filtered to the respective nominal resolution and used to find the coordinates of individual LPOR dimers along the helical path. A total of 20,082 and 17,080 sub-boxes were extracted from particles in classes 1 and 2, respectively, then aligned in Dynamo. For both datasets, a further subtilt refinement step was performed. Using the dyn2rel package ${ }^{66}, 3 \mathrm{D}$ sub-boxes contributing to each map were automatically traced back to their two-dimensional projections, cropped from each image of the tilt series and finally processed with Relion-3 (ref. ${ }^{67}$ ). Both maps attained a final resolution of $9 \AA$.

Structural features were compared to a 3D model generated with I-TASSER ${ }^{36}$ from the primary sequence of $Z$. mays LPOR (GenBank: PWZ38724.1) based on the X-ray structure of Synechocystis LPOR (PDB: 6r48). The 50-amino acid transit peptide at the $\mathrm{N}$ terminus was excluded. All final maps were displayed with ChimeraX ${ }^{68}$

Statistics and reproducibility. Etioplasts from pea and corn were analysed from 28 and 11 independent preparations, respectively. The micrographs, tomographic reconstructions and experimental results in all figures are representative of at least three independent replicates. The tomograms used for subtomogram averaging analysis were collected on grids prepared from three (pea) and five (maize) independent purifications. All attempts to reproduce the results were successful.

Reporting Summary. Further information on research design is available in the Nature Research Reporting Summary linked to this article.

\section{Data availability}

The cryo-EM maps were deposited in the Electron Microscopy Data Bank with accession codes EMD-11959 (ribosome), EMD-11958 (ATP synthase), EMD11961 (LPOR in membrane tubes from class 1), EMD-11960 (LPOR in membrane tubes from class 2), EMD-11963 (LPOR class 1 sub-boxes) and EMD-11962 (LPOR class 2 sub-boxes). Source data are provided with this paper.

Received: 21 August 2020; Accepted: 5 March 2021; Published online: 19 April 2021

\section{References}

1. Daum, B., Nicastro, D., Il, J. A., McIntosh, J. R. \& Kuhlbrandt, W. Arrangement of photosystem II and ATP synthase in chloroplast membranes of spinach and pea. Plant Cell 22, 1299-1312 (2010).

2. Wietrzynski, W. et al. Charting the native architecture of Chlamydomonas thylakoid membranes with single-molecule precision. eLife 9, e53740 (2020).

3. Lindsten, A., Ryberg, M. \& Sundqvist, C. The polypeptide composition of highly purified prolamellar bodies and prothylakoids from wheat (Triticum aestivum) as revealed by silver staining. Physiol. Plant. 72, 167-176 (1988).

4. Philippar, K. et al. Chloroplast biogenesis: the use of mutants to study the etioplast-chloroplast transition. Proc. Natl Acad. Sci. USA 104, 678-683 (2007).

5. Kanervo, E. et al. Expression of protein complexes and individual proteins upon transition of etioplasts to chloroplasts in pea (Pisum sativum). Plant Cell Physiol. 49, 396-410 (2008).

6. Rudowska, L., Gieczewska, K., Mazur, R., Garstka, M. \& Mostowska, A. Chloroplast biogenesis - correlation between structure and function. Biochim. Biophys. Acta Bioenerg. 1817, 1380-1387 (2012).

7. Grzyb, J. M., Solymosi, K., Strzalka, K. \& Mysliwa-Kurdziel, B. Visualization and characterization of prolamellar bodies with atomic force microscopy. J. Plant Physiol. 170, 1217-1227 (2013).

8. Kowalewska, L., Mazur, R., Suski, S., Garstka, M. \& Mostowska, A. Three-dimensional visualization of the tubular-lamellar transformation of the internal plastid membrane network during runner bean chloroplast biogenesis. Plant Cell 28, 875-891 (2016).

9. Adam, Z., Charuvi, D., Tsabari, O., Knopf, R. R. \& Reich, Z. Biogenesis of thylakoid networks in angiosperms: knowns and unknowns. Plant Mol. Biol. 76, 221-234 (2011).

10. Fujii, S., Nagata, N., Masuda, T., Wada, H. \& Kobayashi, K. Galactolipids are essential for internal membrane transformation during etioplast-to-chloroplast differentiation. Plant Cell Physiol. 60, 1224-1238 (2019).

11. Wellburn, A. R., Quail, P. H. \& Gunning, B. E. S. Examination of ribosome-like particles in isolated prolamellar bodies. Planta 134, 45-52 (1977).

12. Ryberg, M., Sandelius, A. S. \& Selstam, E. Lipid-composition of prolamellar bodies and prothylakoids of wheat etioplasts. Physiol. Plant. 57, 555-560 (1983).

13. Sandelius, A. S. \& Selstam, E. Localization of galactolipid biosynthesis in etioplasts isolated from dark-grown wheat (Triticum aestivum L.). Plant Physiol. 76, 1041-1046 (1984).
14. Widellwigge, A. \& Selstam, E. Effects of salt wash on the structure of the prolamellar body membrane and the membrane-binding of nadph-protochlorophyllide oxidoreductase. Physiol. Plant. 78, 315-323 (1990)

15. Selstam, E., Schelin, J., Williams, W. P. \& Brain, A. P. R. Structural organisation of prolamellar bodies (PLB) isolated from Zea mays. Parallel TEM, SAXS and absorption spectra measurements on samples subjected to freeze-thaw, reduced $\mathrm{pH}$ and high-salt perturbation. Biochim. Biophys. Acta Biomembr. 1768, 2235-2245 (2007).

16. Ploscher, M., Reisinger, V. \& Eichacker, L. A. Proteomic comparison of etioplast and chloroplast protein complexes. J. Proteom. 74, 1256-1265 (2011).

17. Shen, Z. et al. Label-free quantitative proteomics analysis of etiolated maize seedling leaves during greening. Mol. Cell Proteomics 8, 2443-2460 (2009).

18. Selstam, E., Widell, A. \& Johansson, L. B. A. A comparison of prolamellar bodies from wheat, Scots pine and Jeffrey pine - pigment spectra and properties of protochlorophyllide oxidoreductase. Physiol. Plant. 70, 209-214 (1987).

19. Blomqvist, L. A., Ryberg, M. \& Sundqvist, C. Proteomic analysis of highly purified prolamellar bodies reveals their significance in chloroplast development. Photosynth. Res. 96, 37-50 (2008).

20. Engdahl, S., Aronsson, H., Sundqvist, C., Timko, M. P. \& Dahlin, C. Association of the NADPH:protochlorophyllide oxidoreductase (POR) with isolated etioplast inner membranes from wheat. Plant J. 27, 297-304 (2001).

21. Zhang, S. et al. Structural basis for enzymatic photocatalysis in chlorophyll biosynthesis. Nature 574, 722-725 (2019).

22. Erdei, N., Barta, C., Hideg, E. \& Boddi, B. Light-induced wilting and its molecular mechanism in epicotyls of dark-germinated pea (Pisum sativum L.) seedlings. Plant Cell Physiol. 46, 185-191 (2005).

23. Masuda, T. \& Fujita, Y. Regulation and evolution of chlorophyll metabolism. Photochem. Photobiol. Sci. 7, 1131-1149 (2008).

24. Buhr, F. et al. Photoprotective role of NADPH:protochlorophyllide oxidoreductase A. Proc. Natl Acad. Sci. USA 105, 12629-12634 (2008).

25. Muraki, N. et al. X-ray crystal structure of the light-independent protochlorophyllide reductase. Nature 465, 110-124 (2010).

26. Heyes, D. J. \& Hunter, C. N. Identification and characterization of the product release steps within the catalytic cycle of protochlorophyllide oxidoreductase. Biochemistry 43, 8265-8271 (2004).

27. Heyes, D. J., Menon, B. R. K., Sakuma, M. \& Scrutton, N. S. Conformational events during ternary enzyme-substrate complex formation are rate limiting in the catalytic cycle of the light-driven enzyme protochlorophyllide oxidoreductase. Biochemistry 47, 10991-10998 (2008).

28. Heyes, D. J., Sakuma, M., de Visser, S. P. \& Scrutton, N. S. Nuclear quantum tunneling in the light-activated enzyme protochlorophyllide oxidoreductase. J. Biol. Chem. 284, 3762-3767 (2009).

29. Reinbothe, C., Buhr, F., Pollmann, S. \& Reinbothe, S. In vitro reconstitution of light-harvesting POR-protochlorophyllide complex with protochlorophyllides $a$ and $b$. J. Biol. Chem. 278, 807-815 (2003).

30. Gabruk, M. et al. Photoactive protochlorophyllide-enzyme complexes reconstituted with PORA, PORB and PORC proteins of $A$. thaliana: fluorescence and catalytic properties. PloS ONE 10, e0116990 (2015).

31. Ryberg, M. \& Sundqvist, C. The regular ultrastructure of isolated prolamellar bodies depends on the presence of membrane-bound NADPH-protochlorophyllide oxidoreductase. Physiol. Plant. 73, 218-226 (1988).

32. Sperling, U., van Cleve, B., Frick, G., Apel, K. \& Armstrong, G. A. Overexpression of light-dependent PORA or PORB in plants depleted of endogenous POR by far-red light enhances seedling survival in white light and protects against photooxidative damage. Plant J. 12, 649-658 (1997)

33. Franck, F. et al. Regulation of etioplast pigment-protein complexes, inner membrane architecture, and protochlorophyllide alpha chemical heterogeneity by light-dependent NADPH:protochlorophyllide oxidoreductases A and B. Plant Physiol. 124, 1678-1696 (2000).

34. Frick, G., Su, Q. X., Apel, K. \& Armstrong, G. A. An Arabidopsis porB porC double mutant lacking light-dependent NADPH:protochlorophyllide oxidoreductases B and C is highly chlorophyll-deficient and developmentally arrested. Plant J. 35, 141-153 (2003).

35. Masuda, T. et al. Functional analysis of isoforms of NADPH: protochlorophyllide oxidoreductase (POR), PORB and PORC, in Arabidopsis thaliana. Plant Cell Physiol. 44, 963-974 (2003).

36. Yang, J. Y. \& Zhang, Y. I-TASSER server: new development for protein structure and function predictions. Nucleic Acids Res. 43, W174-W181 (2015).

37. Kambakam, S., Bhattacharjee, U., Petrich, J. \& Rodermel, S. PTOX mediates novel pathways of electron transport in etioplasts of arabidopsis. Mol. Plant $\mathbf{9}$, 1240-1259 (2016).

38. Renato, M., Boronat, A. \& Azcon-Bieto, J. Respiratory processes in non-photosynthetic plastids. Front. Plant Sci. 6, 496 (2015) 
39. Bieri, P., Leibundgut, M., Saurer, M., Boehringer, D. \& Ban, N. The complete structure of the chloroplast $70 \mathrm{~S}$ ribosome in complex with translation factor pY. EMBO J. 36, 475-486 (2017).

40. Diaconu, M. et al. Structural basis for the function of the ribosomal L7/12 stalk in factor binding and GTPase activation. Cell 121, 991-1004 (2005).

41. Frauenfeld, J. et al. Cryo-EM structure of the ribosome-SecYE complex in the membrane environment. Nat. Struct. Mol. Biol. 18, 614-621 (2011).

42. Yamamoto, H., Kojima-Ando, H., Ohki, K. \& Fujita, Y. Formation of prolamellar-body-like ultrastructures in etiolated cyanobacterial cells overexpressing light-dependent protochlorophyllide oxidoreductase in Leptolyngbya boryana. J. Gen. Appl. Microbiol. 66, 129-139 (2020).

43. Boddi, B., Lindsten, A., Ryberg, M. \& Sundqvist, C. On the aggregational states of protochlorophyllide and its protein complexes in wheat etioplasts. Physiol. Plant. 76, 135-143 (1989).

44. Gabruk, M. et al. Insight into the oligomeric structure of PORA from A. thaliana. Biochim. Biophys. Acta Proteins Proteom. 1864, 1757-1764 (2016).

45. Gabruk, M., Mysliwa-Kurdziel, B. \& Kruk, J. MGDG, PG and SQDG regulate the activity of light-dependent protochlorophyllide oxidoreductase. Biochem. J. 474, 1307-1320 (2017)

46. Reinbothe, C., Lebedev, N. \& Reinbothe, S. A protochlorophyllide light-harvesting complex involved in de-etiolation of higher plants. Nature 397, 80-84 (1999)

47. Reinbothe, C., Lepinat, A., Deckers, M., Beck, E. \& Reinbothe, S. The extra loop distinguishing POR from the structurally related short-chain alcohol dehydrogenases is dispensable for pigment binding but needed for the assembly of light-harvesting POR-protochlorophyllide complex. J. Biol. Chem 278, 816-822 (2003).

48. Zhang, S. et al. Dual role of the active site 'lid' regions of protochlorophyllide oxidoreductase in photocatalysis and plant development. FEBS J. 288, 175-189 (2020).

49. Park, H., Kreunen, S. S., Cuttriss, A. J., DellaPenna, D. \& Pogson, B. J. Identification of the carotenoid isomerase provides insight into carotenoid biosynthesis, prolamellar body formation, and photomorphogenesis. Plant Cell 14, 321-332 (2002).

50. Mysliwa-Kurdziel, B., Kruk, J. \& Strzalka, K. Protochlorophyllide and protochlorophyll in model membranes - an influence of hydrophobic side chain moiety. Biochim. Biophys. Acta Biomembr. 1828, 1075-1082 (2013).

51. Mysliwa-Kurdziel, B., Kruk, J. \& Strzalka, K. Protochlorophyllide in model systems - an approach to in vivo conditions. Biophys. Chem. 175, 28-38 (2013).

52. Pinnola, A. \& Bassi, R. Molecular mechanisms involved in plant photoprotection. Biochem. Soc. Trans. 46, 467-482 (2018)

53. Haniewicz, P. et al. Isolation of monomeric photosystem II that retains the subunit PsbS. Photosynth. Res. 118, 199-207 (2013).

54. Wilkes, M. et al. Molecular insights into lipid-assisted $\mathrm{Ca}^{2+}$ regulation of the TRP channel Polycystin-2. Nat. Struct. Mol. Biol. 24, 123-130 (2017).

55. Haniewicz, P. et al. Isolation of plant photosystem II complexes by fractional solubilization. Front. Plant Sci. 6, 1100 (2015).

56. D'Imprima, E. et al. Protein denaturation at the air-water interface and how to prevent it. eLife 8, e42747 (2019)

57. Hagen, W. J. H., Wan, W. \& Briggs, J. A. G. Implementation of a cryo-electron tomography tilt-scheme optimized for high resolution subtomogram averaging. J. Struct. Biol. 197, 191-198 (2017).

58. Mastronarde, D. N. Automated electron microscope tomography using robust prediction of specimen movements. J. Struct. Biol. 152, 36-51 (2005).

59. Zheng, S. Q. et al. MotionCor2: anisotropic correction of beam-induced motion for improved cryo-electron microscopy. Nat. Methods 14, 331-332 (2017).

60. Kremer, J. R., Mastronarde, D. N. \& McIntosh, J. R. Computer visualization of three-dimensional image data using IMOD. J. Struct. Biol. 116, 71-76 (1996)

61. Frangakis, A. S. \& Hegerl, R. Noise reduction in electron tomographic reconstructions using nonlinear anisotropic diffusion. J. Struct. Biol. 135, 239-250 (2001).
62. Chen, M. et al. A complete data processing workflow for cryo-ET and subtomogram averaging. Nat. Methods 16, 1161-1168 (2019).

63. Castano-Diez, D. The Dynamo package for tomography and subtomogram averaging: components for MATLAB, GPU computing and EC2 Amazon Web Services. Acta Crystallogr. D 73, 478-487 (2017).

64. Scheres, S. H. \& Chen, S. Prevention of overfitting in cryo-EM structure determination. Nat. Methods 9, 853-854 (2012).

65. Nicastro, D. et al. The molecular architecture of axonemes revealed by cryoelectron tomography. Science 313, 944-948 (2006).

66. Sanchez, R. M., Zhang, Y., Chen, W., Dietrich, L. \& Kudryashev, M. Subnanometer-resolution structure determination in situ by hybrid subtomogram averaging - single particle cryo-EM. Nat. Commun. 11, 3709 (2020)

67. Zivanov, J. et al. New tools for automated high-resolution cryo-EM structure determination in RELION-3. elife 7, e42166 (2018).

68. Goddard, T. D. et al. UCSF ChimeraX: meeting modern challenges in visualization and analysis. Protein Sci. 27, 14-25 (2018).

\section{Acknowledgements}

We thank M. Linder for expert assistance in performing the immuno-gold labelling experiment; B. Daum for invaluable advice in the early stages of project design; S. Welsch, D. J. Mills, S. Prinz, M. Linder and S. Kaltwasser for EM support; C. Kunz for laboratory support; and R. Sanchez, L. Dietrich and Y. Zhang for useful discussions about tomographic data acquisition and processing.

\section{Author contributions}

D.F. conceived, designed and executed the project; managed the growth of plant specimens; wet-lab experiments; cryo-EM specimen preparation; cryo-EM data collection, reconstruction, analysis and validation; and co-wrote the manuscript. W.K. conceived and supervised the project; managed resources and funding acquisition; and co-wrote the manuscript.

\section{Competing interests}

The authors declare no competing interests.

\section{Additional information}

Extended data is available for this paper at https://doi.org/10.1038/s41477-021-00896-z.

Supplementary information The online version contains supplementary material available at https://doi.org/10.1038/s41477-021-00896-z.

Correspondence and requests for materials should be addressed to D.F. or W.K. Peer review information Nature Plants thanks Benjamin Engel and the other, anonymous, reviewer(s) for their contribution to the peer review of this work.

Reprints and permissions information is available at www.nature.com/reprints. Publisher's note Springer Nature remains neutral with regard to jurisdictional claims in published maps and institutional affiliations.

Open Access This article is licensed under a Creative Commons Attribution 4.0 International License, which permits use, sharing, adap tation, distribution and reproduction in any medium or format, as long as you give appropriate credit to the original author(s) and the source, provide a link to the Creative Commons license, and indicate if changes were made. The images or other third party material in this article are included in the article's Creative Commons license, unless indicated otherwise in a credit line to the material. If material is not included in the article's Creative Commons license and your intended use is not permitted by statutory regulation or exceeds the permitted use, you will need to obtain permission directly from the copyright holder. To view a copy of this license, visit http://creativecommons. org/licenses/by/4.0/.

(c) The Author(s), 2021 
a

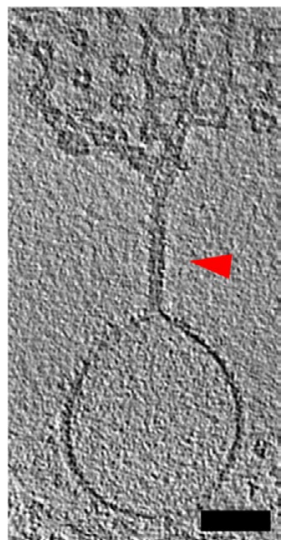

C

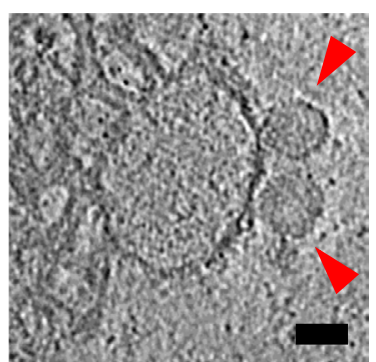

b

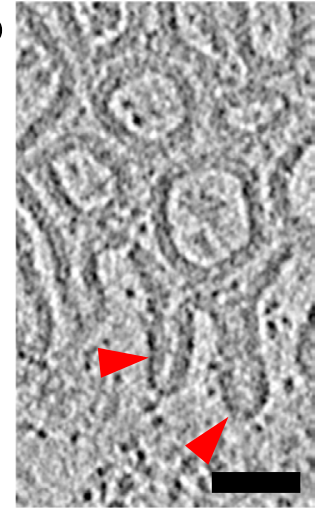

d

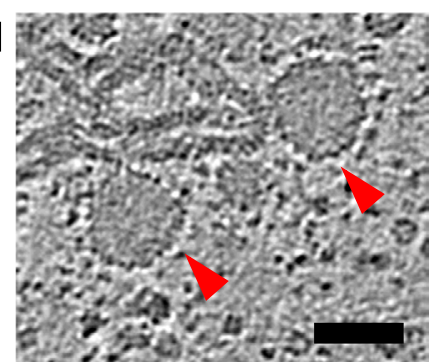

e

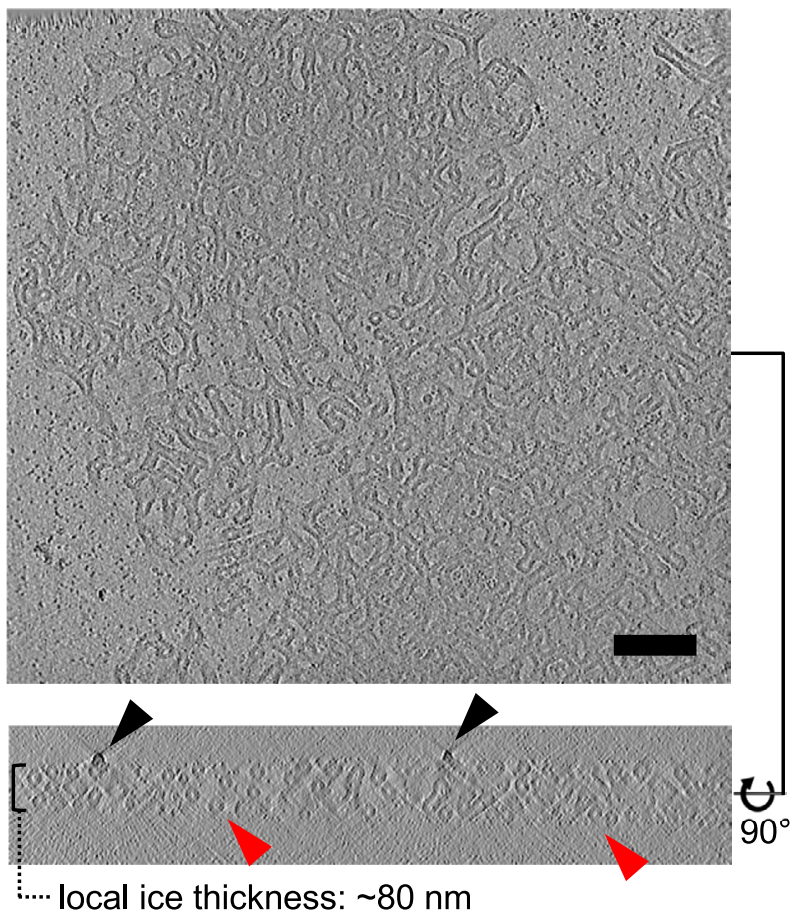

Extended Data Fig. 1 | Cryo-tomography of pea etioplast inner membranes. a, Partly detached prothylakoid connected to the prolamellar body by a membrane tube (red arrowhead). b, Sealed free ends of the tubular mesh (red arrowheads). c, Plastoglobuli (red arrowheads) attached to prothylakoids and (d) tubular membranes. e, Disordered pea prolamellar body squeezed into a thin slab of vitrified buffer. The circular profile of multiple membrane tubes is visible in tomographic slices at a $90^{\circ}$ angle to the grid plane (red arrowheads). Atmospheric ice contamination (black arrowheads) delineates the air-water interface. All images are slices of tomographic volumes. Scale bar size: $100 \mathrm{~nm}(\mathrm{a}), 50 \mathrm{~nm}$ (b, c, d), $150 \mathrm{~nm}(\mathrm{e})$. 


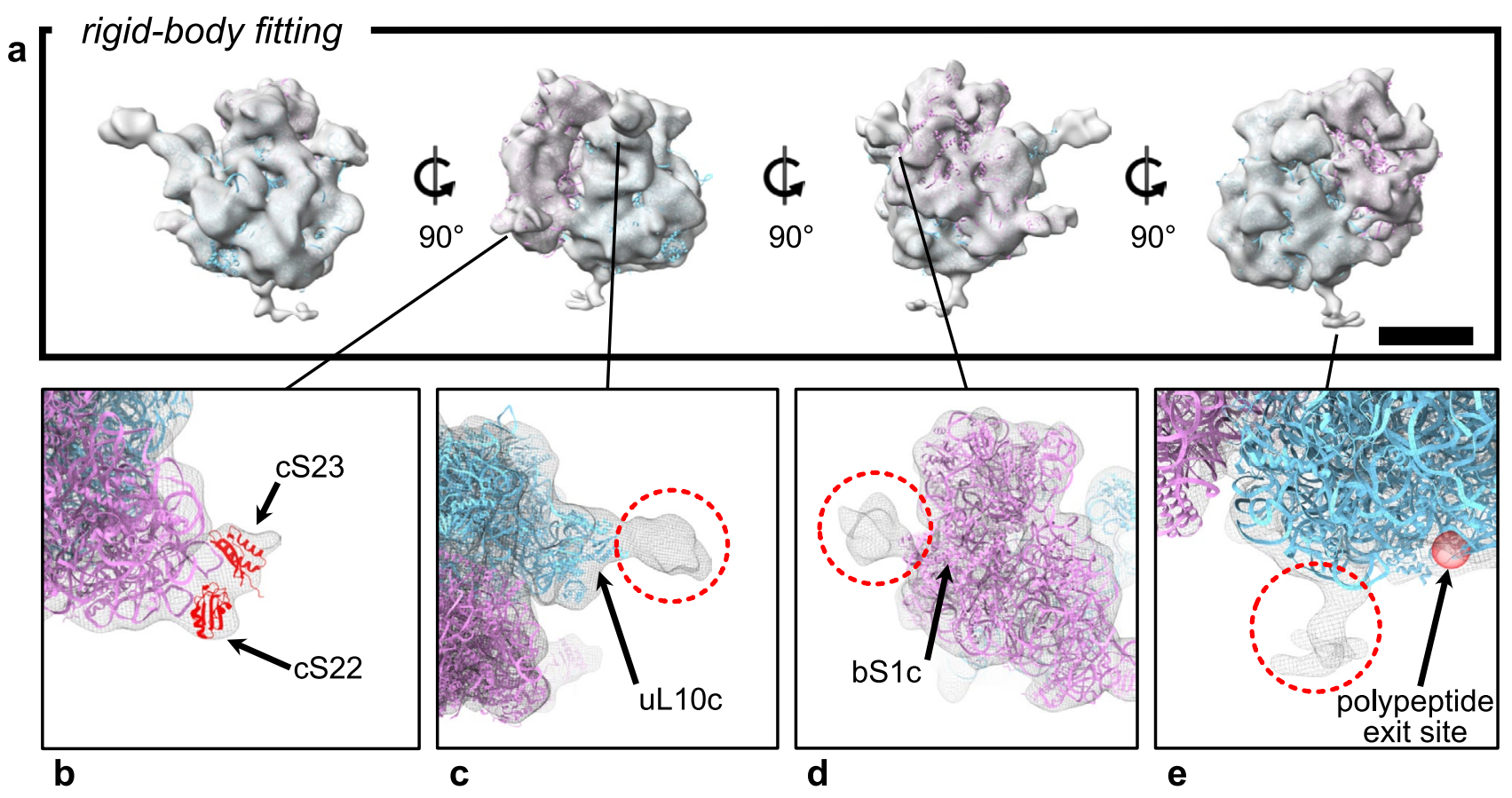

Extended Data Fig. 2 | Chloroplast ribosomes in the prolamellar body. (a) Rigid-body fitting shows good correspondence between subtomogram average and atomic model of the 5OS (PDB 5mmi, cyan) and 30S (PDB 5mmj, pink) subunit of the chloroplast ribosome. Inset (b) highlights the position of plastid-specific subunits cS22 and cS23 (red) and their fit in the foot region of the density map (grey mesh). Inset (c) and (d) indicate the unassigned densities (dashed red circles) connected to the uL10c and bS1c subunits. Inset (e) shows the location of the polypeptide exit site (red sphere), and the unassigned density (dashed red circle) next to it. Scale bar size: $10 \mathrm{~nm}$. 


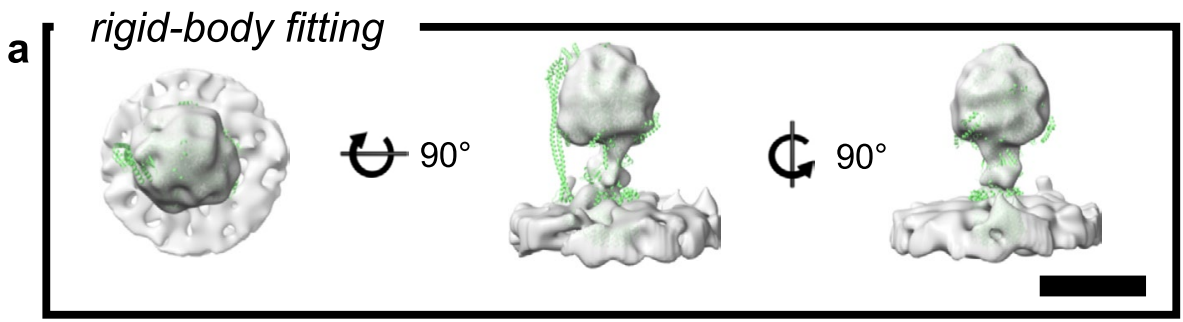

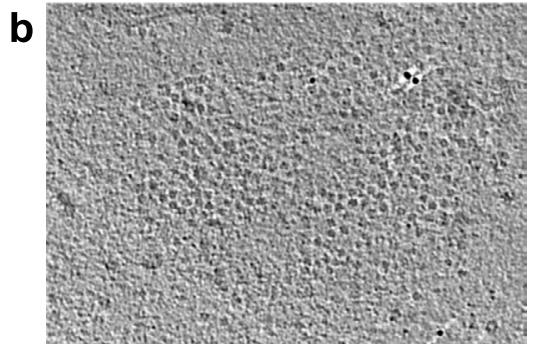

\#1

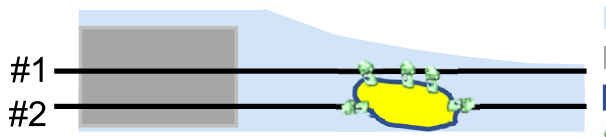

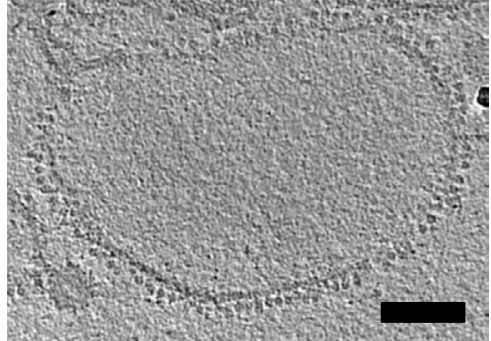

\#2

vitrified buffer

holey carbon

prothylakoid

ATP synthase

C

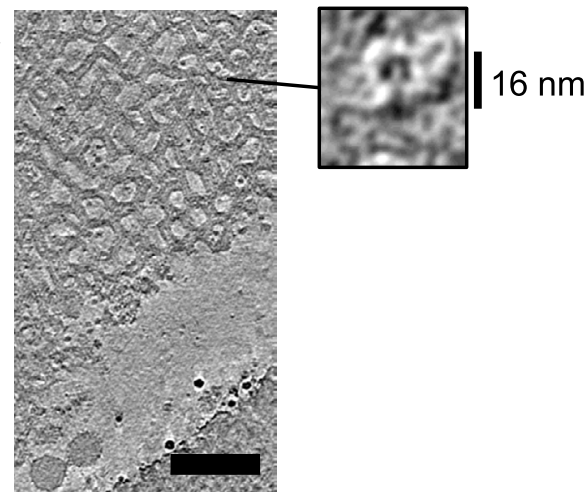

Extended Data Fig. 3 | Structure and distribution of ATP synthase in prothylakoids. a, Rigid-body fitting of chloroplast ATP synthase (PDB 6fkf, green) to subtomogram average map shows good correspondence with the F1 head and central stalk, while the peripheral stalk is not resolved. b, Tomographic slices through the surface (\#1) and centre (\#2) of a prothylakoid vesicle indicate random distribution of ATP synthase monomers. Similar densities are occasionally found in prolamellar bodies (c). Scale bar size: 10 nm (a), 100 nm (b), 150 nm (c). 


\section{NATURE PLANTS}

ARTICLES

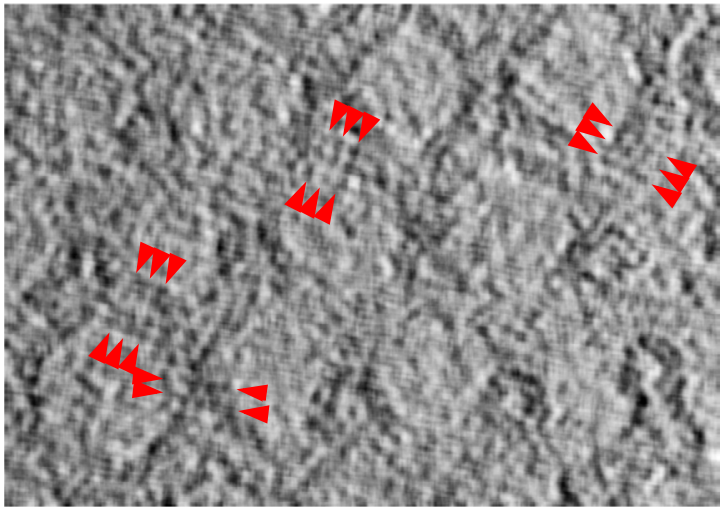

\#1

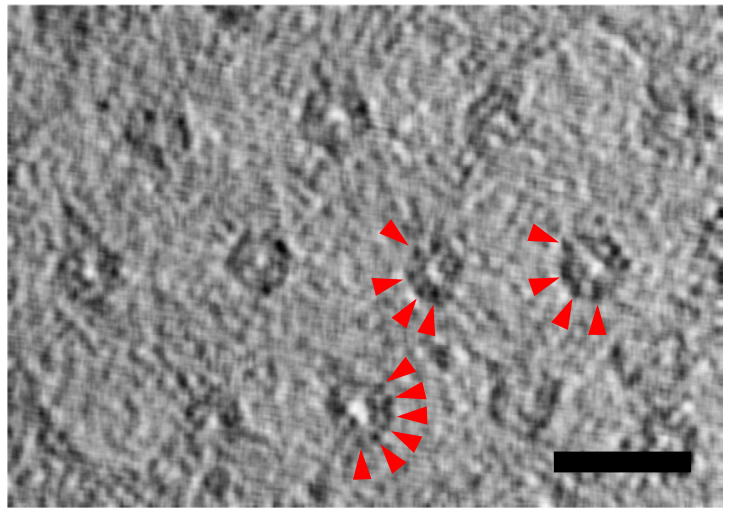

\#2

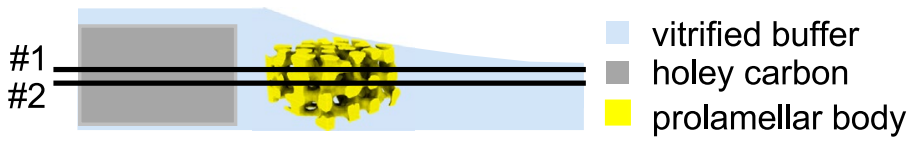

Extended Data Fig. 4 | Features of tetrahedrally branched membrane tubes. Slices through the surface of the membrane tubes (\#1) show seemingly regular arrays of small densities (highlighted between red arrowheads) when visualized in the unbinned tomographic reconstruction of the same ordered region shown in Fig. 1e. In cross-section (\#2), the same densities are partly resolved along the circumference of the membrane tube (red arrowheads). Scale bar size: $60 \mathrm{~nm}$. 


\section{Low magnification overview}

a

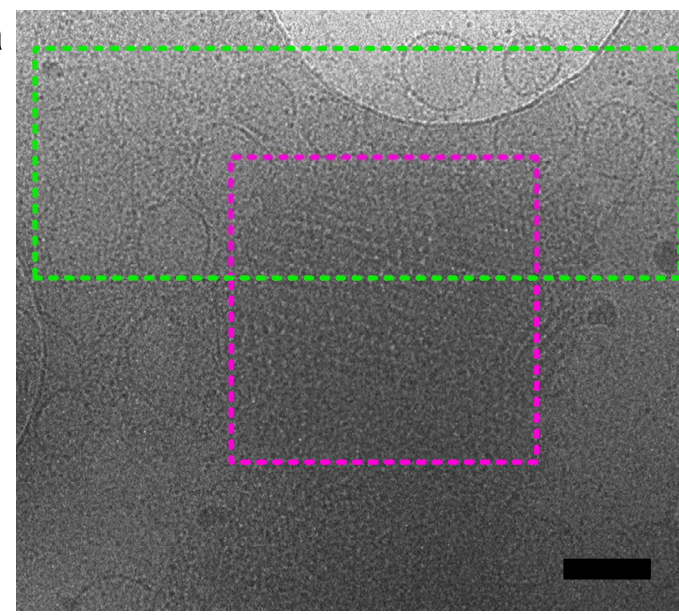

Section through tomographic reconstructions

e

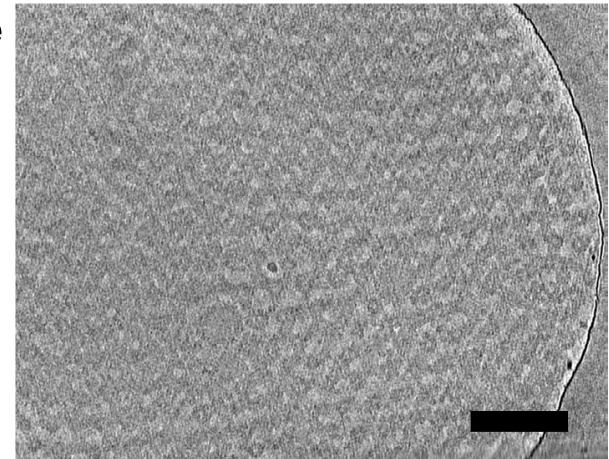

(local ice thickness: $\sim 340 \mathrm{~nm}$ ) b

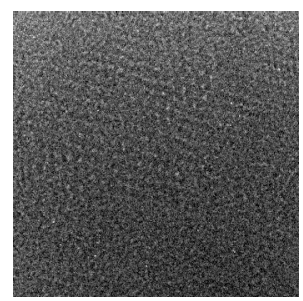

c

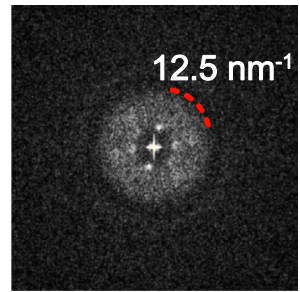

d

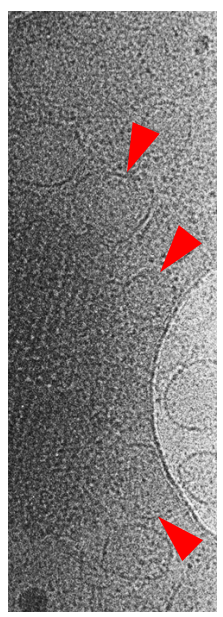

f

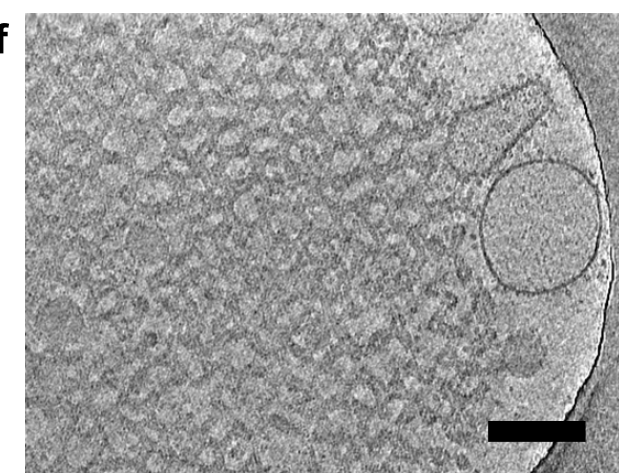

(local ice thickness: $\sim 220 \mathrm{~nm}$ )

Extended Data Fig. $\mathbf{5}$ | Inner membrane of maize etioplasts. (a) Low-magnification view of vitrified inner membranes. Isolated patches of the same micrograph (dashed magenta square and green rectangle) highlight the morphology of prolamellar bodies (b, and its Fourier transform, $\mathbf{c}$ ) and prothylakoids (d, red arrowheads). Structural features closely resemble those in pea (see Fig. 1a-d). Prolamellar bodies of maize etioplasts are regular (e) and likewise have prothylakoids at their periphery ( $\mathbf{f}$ and red arrowheads in d). Scale bar size: $250 \mathrm{~nm}$ (a), $150 \mathrm{~nm}$ (e, f). 
a

sucrose gradient

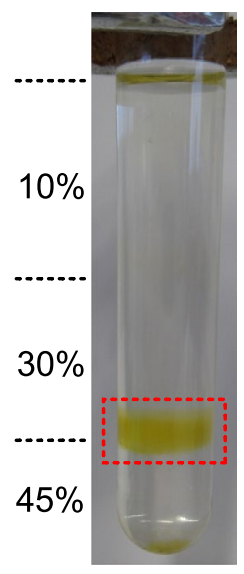

b

SDS-PAGE and western blot

M

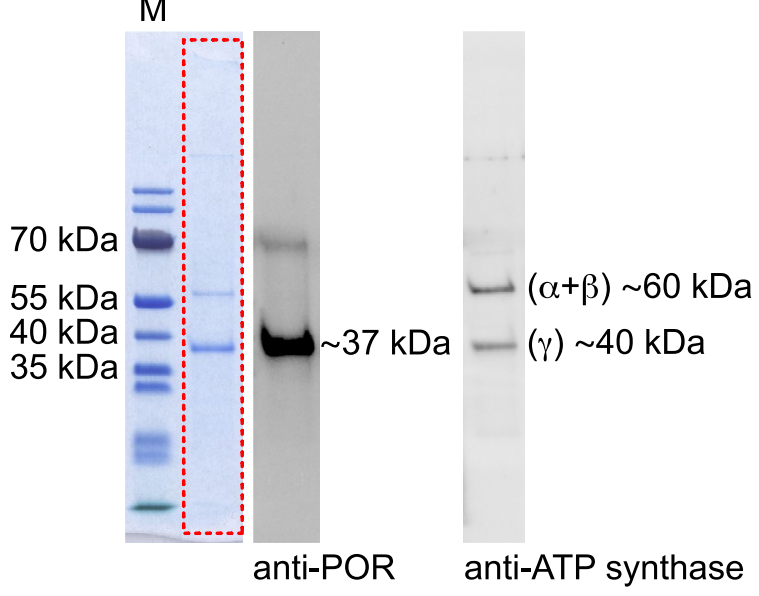

C immuno-gold labelling (leaf plastic section)

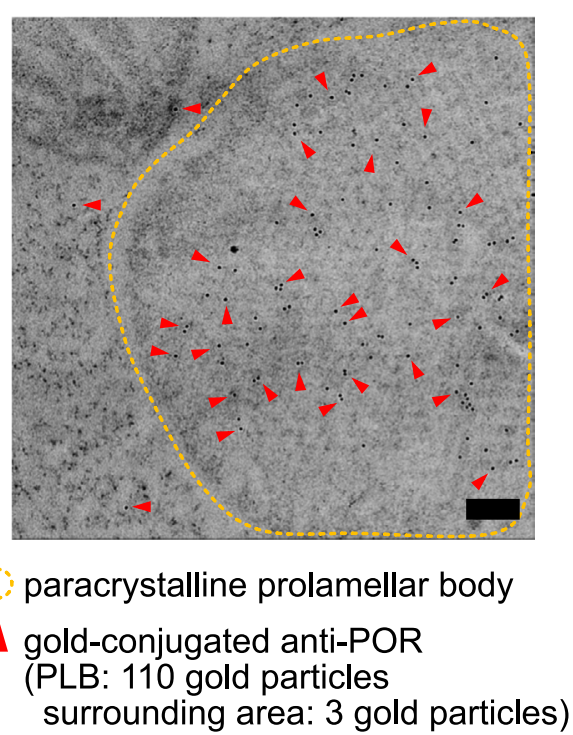

Extended Data Fig. 6 | Protein composition of etioplast inner membranes. a, Inner membrane fraction at the 30-45\% interface (red dashed rectangle) of a sucrose gradient. b, SDS-PAGE of the same fraction indicates a prominent band around $35-40 \mathrm{kDa}$, and a second band above $55 \mathrm{kDa}$, identified respectively as POR and ATP synthase subunits by Western blot analysis. M, molecular markers. c, Gold-conjugated anti-POR antibodies (red arrowheads) decorate the paracrystalline prolamellar body (orange dashed line). Unspecific labelling is negligible. Scale bar size: $250 \mathrm{~nm}$. 
a

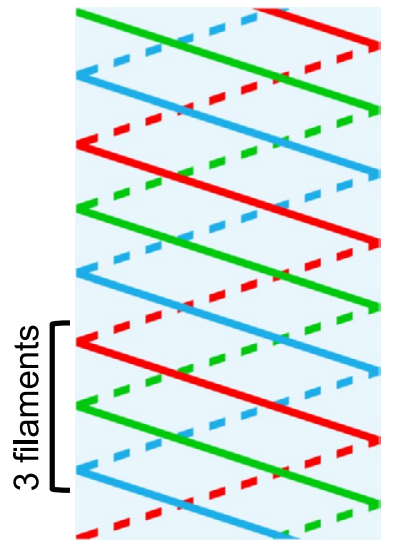

3-start helix b

class 1

(16 units per turn)

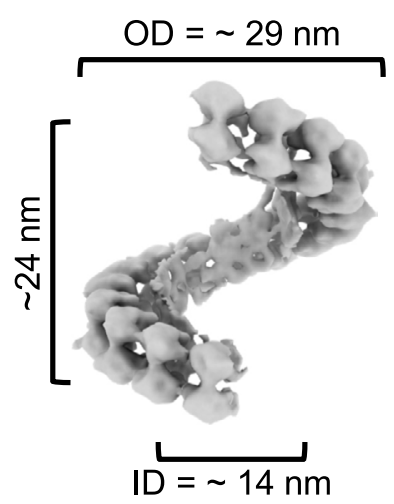

class 2

(15 units per turn)

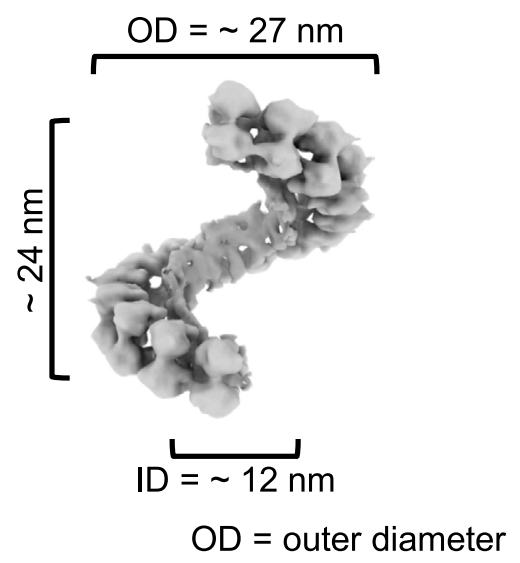

ID = inner lumenal diameter

Extended Data Fig. 7 | Details of helical arrays. a, Graphical representation of a 3-start helix. b, Size comparison between single helix turns of class 1 and class 2 subtomogram averages (Fig. 3c). 
class 1 subboxes

(16 units per turn)

$x$
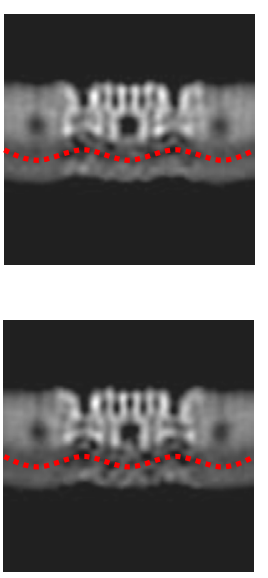

Y
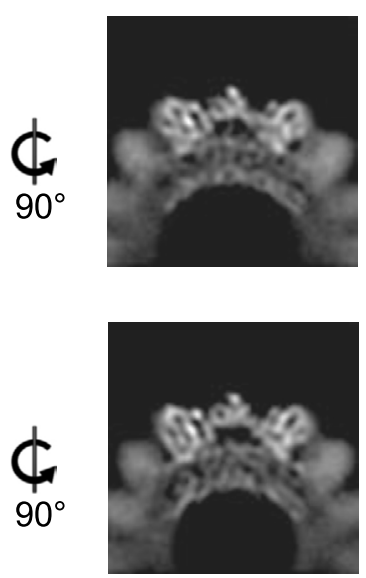

Z

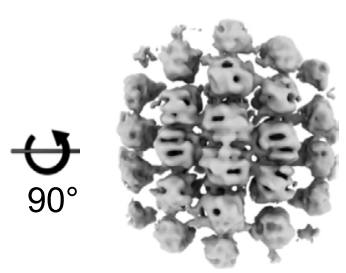

$\theta$

$90^{\circ}$

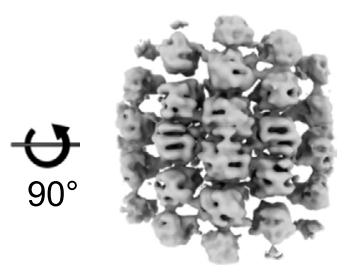

class 2 subboxes (15 units per turn)

Extended Data Fig. 8 | Comparison of class 1 and class 2 subboxes. Central section of side views ( $X$ and $Y$ ), and isosurface of top view ( $Z$ ) of subtomogram averages from class 1 and 2 subboxes. Dashed red lines trace the outer membrane surface, highlighting negative membrane curvature below each dimer. Scale bar size: $80 \AA$. 


\section{ARTICLES}

a

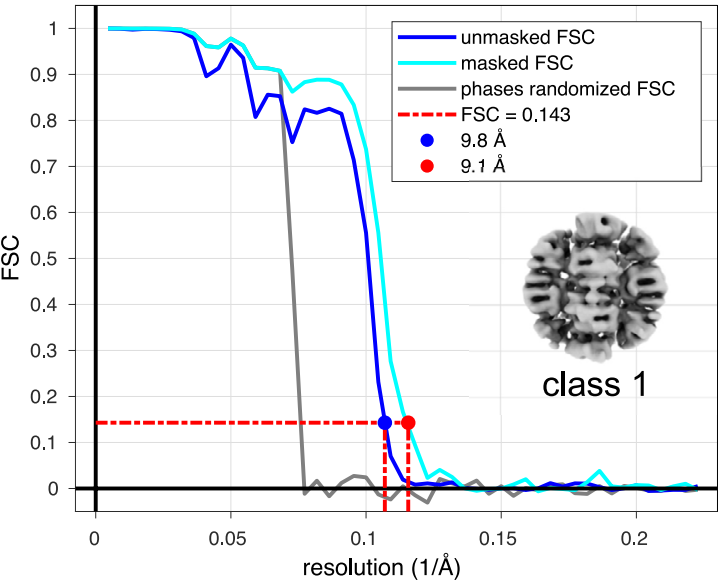

b

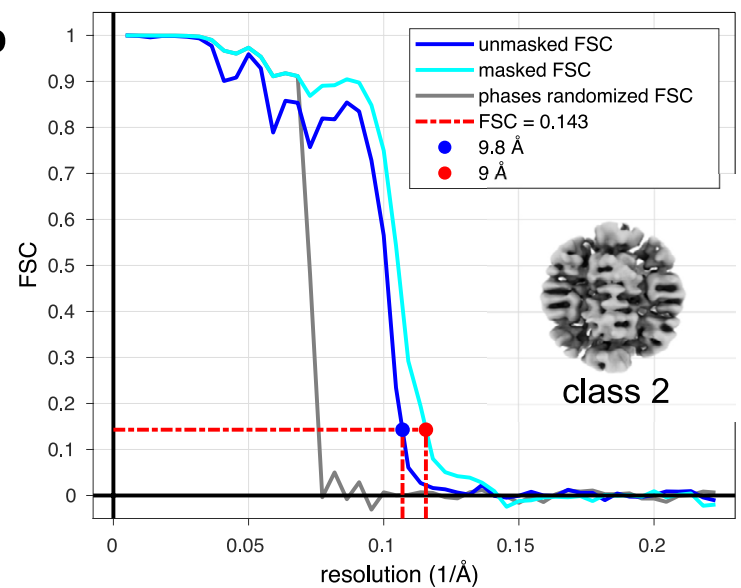

Extended Data Fig. 9 | Resolution of subtomogram averages of membrane tube subboxes. Fourier shell correlation of unfiltered half-maps from class 1 (a) and class 2 (b). Map resolutions are $9.1 \AA$ (class 1 ), and $9 \AA$ (class 2 ). 
a
LPOR Zea mays
(I-TASSER prediction model) this work

b

\section{LPOR Synechocystis} (X-ray structure, PDB 6R48) Zhang et al. 2019

C

superposition
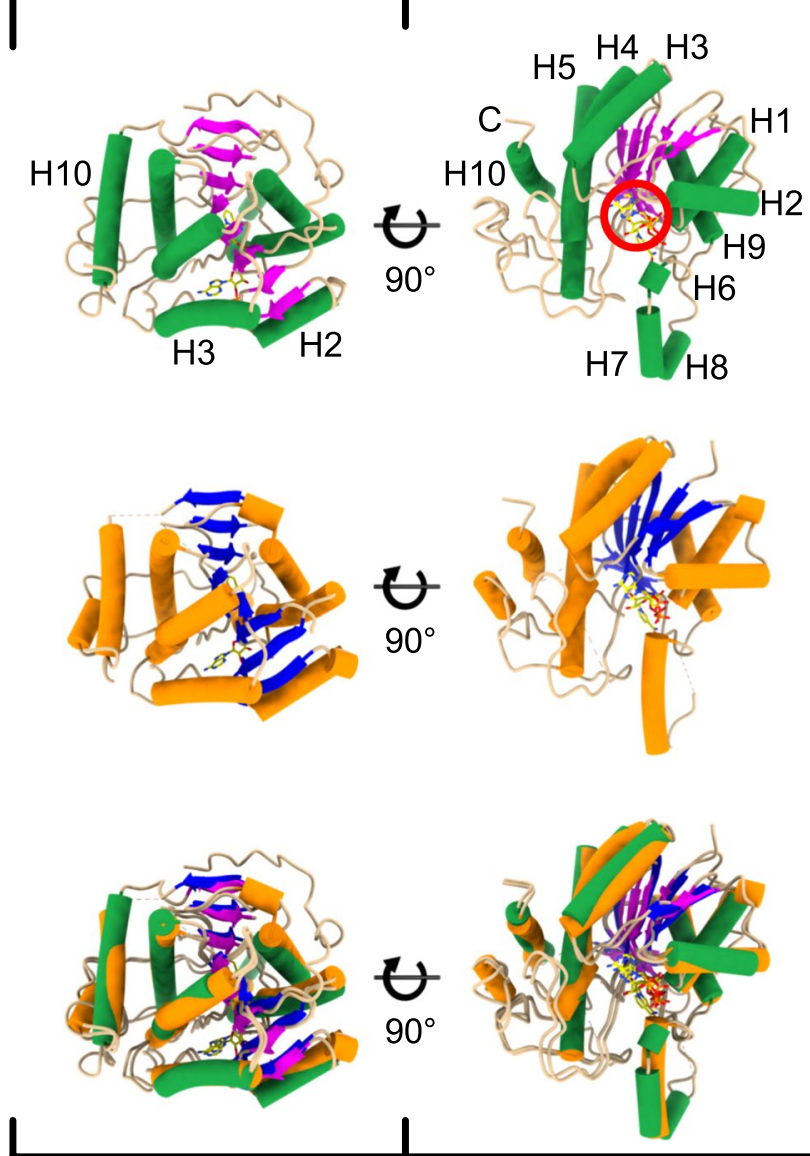

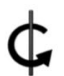
$90^{\circ}$
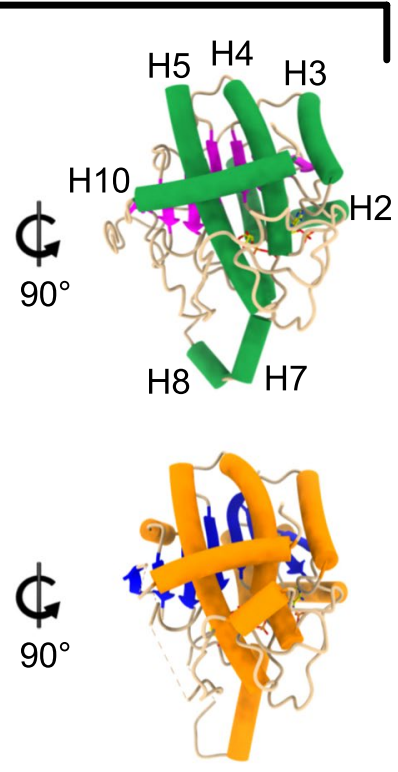

side views

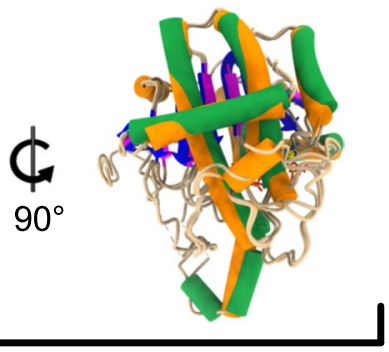

Extended Data Fig. 10 | Atomic models of LPOR. Maize model generated by I-TASSER fitted to the subtomogram average volume (a) and X-ray structure from Synechocystis (b) agree closely (c). 


\section{Reporting Summary}

Nature Research wishes to improve the reproducibility of the work that we publish. This form provides structure for consistency and transparency in reporting. For further information on Nature Research policies, see our Editorial Policies and the Editorial Policy Checklist.

\section{Statistics}

For all statistical analyses, confirm that the following items are present in the figure legend, table legend, main text, or Methods section.

n/a Confirmed

$\square \bigotimes$ The exact sample size $(n)$ for each experimental group/condition, given as a discrete number and unit of measurement

$\square$ \A statement on whether measurements were taken from distinct samples or whether the same sample was measured repeatedly

$\triangle$ The statistical test(s) used AND whether they are one- or two-sided

Х Only common tests should be described solely by name; describe more complex techniques in the Methods section.

Х $\square$ A description of all covariates tested

$\bigotimes \square$ A description of any assumptions or corrections, such as tests of normality and adjustment for multiple comparisons

$\square$ A full description of the statistical parameters including central tendency (e.g. means) or other basic estimates (e.g. regression coefficient)

\ $\square$ AND variation (e.g. standard deviation) or associated estimates of uncertainty (e.g. confidence intervals)

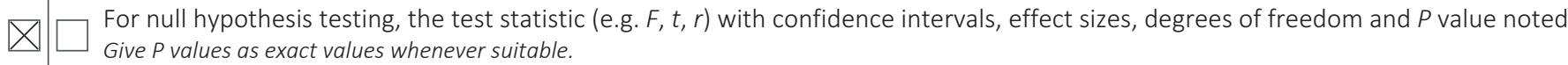

Х $\square$ For Bayesian analysis, information on the choice of priors and Markov chain Monte Carlo settings

Х $\square$ For hierarchical and complex designs, identification of the appropriate level for tests and full reporting of outcomes

Х $\square$ Estimates of effect sizes (e.g. Cohen's $d$, Pearson's $r$ ), indicating how they were calculated

Our web collection on statistics for biologists contains articles on many of the points above.

\section{Software and code}

Policy information about availability of computer code

Data collection SerialEM v3.6 (described in: Mastronarde, D. N. 2005. J Struct Biol, 152: 36-51).

Data analysis Dynamo v1.1.253 (described in: Castano-Diez, D. 2017. Acta Crystallogr D Struct Biol, 73: 478-87).

dyn2rel package (described in: Sanchez, R. M., et al. 2020. Nat Commun, 11: 3709).

MATLAB VR2019a (The MathWorks Inc.)

MotionCor2 v1.2.6 (described in: Zheng, S. Q., et al. 2017. Nature Methods, 14: 331-32).

IMOD v4.8.56 (described in: Kremer, J. R., et al. 1996. Journal of Structural Biology, 116: 71-76).

EMAN v2.31 (described in: Chen, M., et al. 2019. Nature Methods, 16: 1161-+).

PEET v1.10.0 (described in: Nicastro, D., et al. 2006. Science, 313: 944-8).

RELION v3.1.0 (described in: Zivanov, J., et al. 2018. Elife, 7).

I-TASSER (described in: Yang, J. Y., and Y. Zhang. 2015. Nucleic Acids Research, 43: W174-W81).

ChimeraX v0.93 (described in: Goddard, T. D. et al. 2018. Protein Sci, 27, 14-25) 
Policy information about availability of data

All manuscripts must include a data availability statement. This statement should provide the following information, where applicable:

- Accession codes, unique identifiers, or web links for publicly available datasets

- A list of figures that have associated raw data

- A description of any restrictions on data availability

All subtomogram averaging maps were deposited in the Electron Microscopy Data Bank (EMDB) with accession code: EMD-11959 (ribosome), EMD-11958 (ATP synthase), EMD-11961 (LPOR in membrane tubes from class 1), EMD-11960 (LPOR in membrane tubes from class 2), EMD-11963 (LPOR class 1 subboxes), and EMD-11962 (LPOR class 2 subboxes).

\section{Field-specific reporting}

Please select the one below that is the best fit for your research. If you are not sure, read the appropriate sections before making your selection. $\bigotimes$ Life sciences Behavioural \& social sciences Ecological, evolutionary \& environmental sciences

For a reference copy of the document with all sections, see nature.com/documents/nr-reporting-summary-flat.pdf

\section{Life sciences study design}

All studies must disclose on these points even when the disclosure is negative.

Sample size Etioplasts from pea and maize were analysed from 28 and 11 independent preparations respectively. 7 tomograms were used for subtomogram averaging of ribosomes and ATP synthase, 17 for subtomogram averaging of LPOR. The number of particles was sufficient for averaging, and confirmed the reproducibility of the results.

Data exclusions 65 tomograms were reconstructed, but only 24 were used for subtomogram averaging. The selection was based on: i) quality of tilt series alignment, assessed with a standard procedure through IMOD's alignment score; ii) specimen thickness, assessed by visual inspection of the tomogram.

Replication The micrographs, tomographic reconstructions, and experimental results in all figures are representative of at least three independent replicates. The tomograms used for subtomogram averaging analysis were collected on grids prepared from 3 (pea) and 5 (corn) independent purifications. Each one of these tomograms was collected on a distinct etioplast, and thus is a biological replicate. All attempts to reproduce the results were successful.

Randomization Etioplast inner membranes on the cryo-EM grid were selected randomly for data acquisition.

Blinding This work is not a case-control study, blinding is not relevant.

\section{Reporting for specific materials, systems and methods}

We require information from authors about some types of materials, experimental systems and methods used in many studies. Here, indicate whether each material, system or method listed is relevant to your study. If you are not sure if a list item applies to your research, read the appropriate section before selecting a response.

Materials \& experimental systems

\begin{tabular}{l|l}
\multicolumn{2}{l}{ Methods } \\
\hline n/a & Involved in the study \\
$\square$ & $\square$ ChIP-seq \\
$\searrow$ & $\square$ Flow cytometry \\
$\square$ & $\square$ MRI-based neuroimaging
\end{tabular}

\section{n/a}

n/a $\mid$ Involved in the study

$\square$ \ Antibodies

$\bigotimes \square$ Eukaryotic cell lines

\ $\square$ Palaeontology and archaeology

Х $\square$ Animals and other organisms

\ $\square$ Human research participants

\ $\square$ Clinical data

$\bigotimes \square$ Dual use research of concern

\section{Antibodies}

Antibodies used

Agrisera (Vännäs, Sweden): Anti-POR (AS05 067) and Anti-ATP synthase (AS08 370) SouthernBiotech (Birmingham (AL), U.S.A.): Goat anti-rabbit secondary antibody (4055-05) Jackson ImmunoResearch Europe Ltd (Ely, UK): gold-conjugated AffiniPure goat anti-rabbit secondary antibody (111-205-144) 
Anti-POR (AS05 067), tested for western blot, confirmed reactivity in A. thaliana, Cyanobacteria, H. vulgare, N. tabacum, O. sativa, P. Sogo Yukidian cultivar V3, P. sativum, T. aestivum. No exceptions from predicted reactivity were confirmed.

Related publications: Dogra et al. (2019), doi: 10.1038/s41467-019-10760-6; Zhang et al. (2018), doi: 10.1016/j.niox.2018.03.001; Han et al. (2015), doi: 10.1111/tpj.12889.

(source: www.agrisera.com)

Anti-ATP synthase (AS08 370), tested for western blot, confirmed reactivity in A. thaliana, C. reinhardtii, S. oleracea, P. sativum, E. ophiuriudes, P. vaginatum. No exceptions from predicted reactivity were confirmed. Additional notes: The prominent reaction is to a doublet around 55-58 kDa which is alpha and beta and weaker reaction to gamma subunit which is around $38 \mathrm{kDa}$.

Related publications: Du et al. (2018), doi: 10.1105/tpc.17.00446; Liu et al. (2011), doi: 10.1016/j.jplph.2011.08.019.

(source: www.southernbiotech.com):

Goat anti-rabbit secondary antibody (4055-05), tested for western blot, confirmed reactivity against rabbit IgG.

Related publications: Wu and Ma (2020), doi: 10.1016/j.archoralbio.2020.104930

(source: www.jacksonimmuno.com, www.citeab.com)

gold-conjugated AffiniPure goat anti-rabbit secondary antibody (111-205-144), tested for immuno-electron microscopy, confirmed reactivity against rabbit lgG.

Related publications: Hamada et al. (2018), doi:10.1242/jcs.216051; Mariotti et al. (2018), doi: 10.1038/s41467-017-02642-6; Farley et al. (2015), doi: 10.1016/j.neuroscience.2015.07.062 